Portland State University

PDXScholar

10-1-1969

\title{
A theoretical and experimental study of the hyperbolic electron mirror
}

Michael Stewart Mauck
Portland State University

Follow this and additional works at: https://pdxscholar.library.pdx.edu/open_access_etds Let us know how access to this document benefits you.

\section{Recommended Citation}

Mauck, Michael Stewart, "A theoretical and experimental study of the hyperbolic electron mirror" (1969). Dissertations and Theses. Paper 940.

https://doi.org/10.15760/etd.940

This Thesis is brought to you for free and open access. It has been accepted for inclusion in Dissertations and Theses by an authorized administrator of PDXScholar. Please contact us if we can make this document more accessible: pdxscholar@pdx.edu. 
AN ABSTRACT OF THE THESIS OF Michael Stewart Mauck for the Master of Science in Physics presented October 17, 1969.

Title: A Theoretical and Experimental Study of the Hyperbolic Electron Mirror.

APPROVED BY MEMBERS OF THE THESIS COMMITTEE:

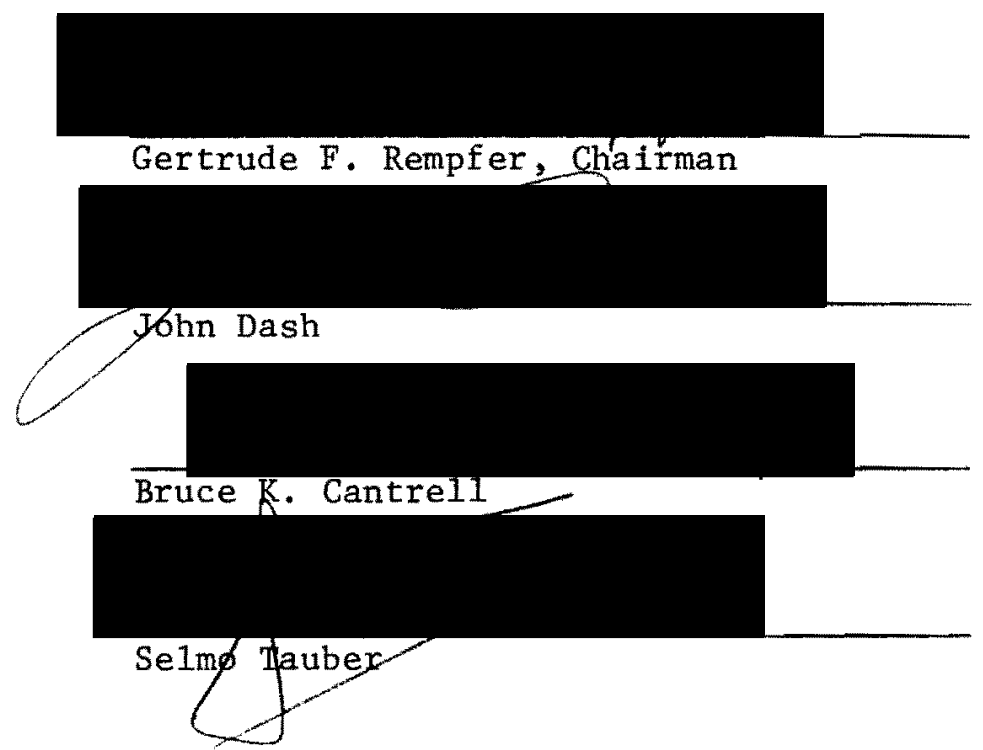

A study of the hyperbolic electrostatic electron mirror is presented. The focal properties of the mirror are determined both theoretically and experimentally. In the theoretical study the mirror field is formed between a positive hyperbolic potential surface and the negative asymptotic cone of half angle arctan $\sqrt{2}$. The focal properties are obtained from the solutions of the equations for the radial and axial motions of the electron in the 
mirror field. The analysis includes the effect of an axial aperture (for entrance and exit of electrons) in the positive surface. The paraxial values of focal length and focal distance and their second order spherical aberrations are calculated by expanding the expressions for the focal properties in terms of the incident radial height. The mirror field in the experimental study is formed between a similar negative electrode and a flat apertured positive electrode. The experimental values of the focal properties are determined by a ray tracing method in which the pattern of shadows cast by a grid in the incident and reflected beams is analyzed. Comparison of the experimentally measured and the theoretically calculated focal properties shows satisfactory agreement. The mirror exhibits over-corrected spherical aberration in certain ranges of its operation which suggests Its use to improve the resolution limit of electron optical devices. 
A THEORETTCLT, AND EXPERTMENTAI, STUDY OF

THE HYPLRBOLTC ELECTRON MIRROR

by

MICHAEL STEWART MAUCK

A thesis submitted in partial fulfillment of the requirements for the degree of

\author{
MASTER OF SCIENCE \\ in \\ PHYSICS
}

Portlanc' Stale Iniversity

1969 
TO THE OFFICE OF GRADUATE STUDIES:

The members of the Committee approve the thesis of

Michae1 Stewart Mauck presented October 17, 1969.

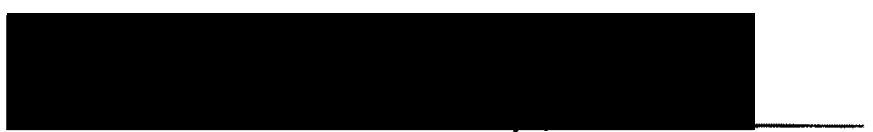

Gertrude F. Rempfer, Chairman

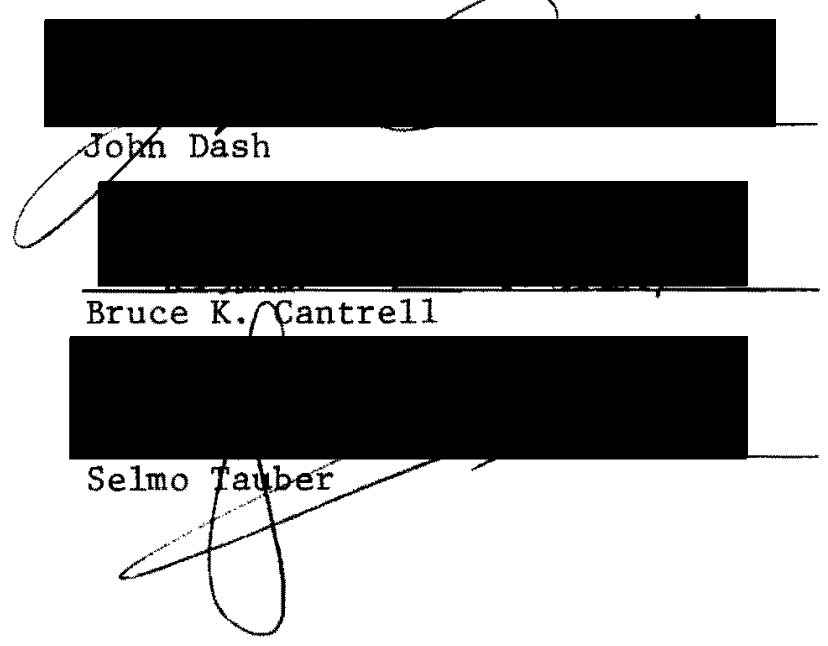

APPROVED :
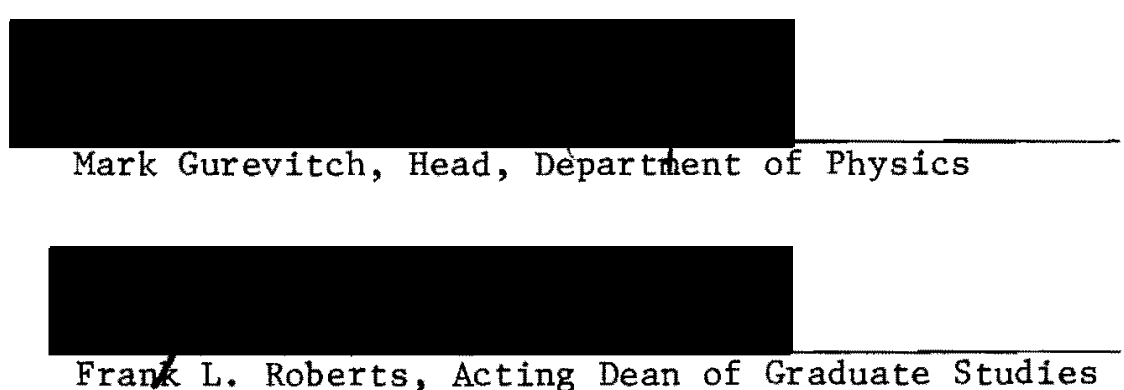
TABT $E_{\text {E }}$ OF CONTENTS

PAGE

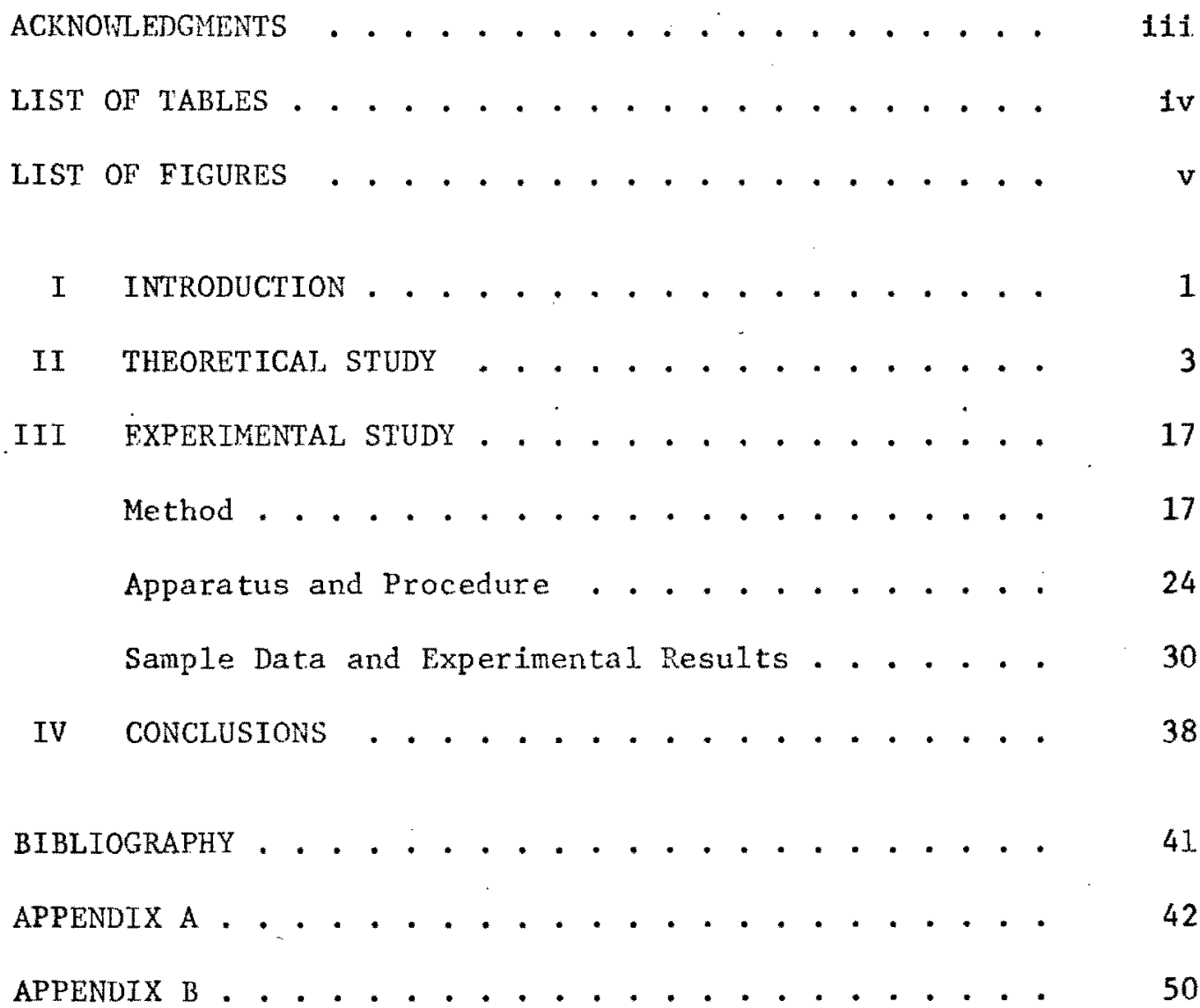




\section{ACKNOWI,FDGHENTS}

The author is deeply indebted to Dr. Gertrude Rempfer for the contents of this paper and wishes to thank her for her unselfish generosity of time and counsel. The author also wishes to thank Tektronix, Inc., for both material and financial support, Bert Cathery for his permission to use the facilities of the Electron Microscope Group, Dr. Selmo Tauber for constructive suggestions and encouragement, Dr. John Dash and Dr. Bruce Cantrell for their useful criticisms. 
I.IST OF TABL.FS

TABLE

PAGE

I Theoretical Results . . . . . . . . . . 16

II Data From a Shadow Pattern . . . . . . . . . 32

III Experimental Results . . . . . . . . . . 37 
LTSI OF ETGURES

FIGURE

PAGE

1 Mirror field used in the experimental study . . . . . 4

2. Mirror field used in the theoretical study . . . . . 4

3 Deflection of an incident ray by the aperture lens . . . 11

4 Deflection of a reflected ray by the aperture lens . . . 11

5 Focal length and focal distance of the mirror . . . . 14

6 Experimental mirror arrangement . . . . . . . . 18

7 Equivalent optical system and ray tracing method . . . . 19

8 Undercorrected geometrical aberrations for para1le1 Incidence .................. 22

9 Photograph of film holder, mirror assembly, and grating 25

10 Photograph of experimental setup . . . . . . . . . 27

11 Electrical circuit . . . . . . . . . . . 28

12 Drawing of the mirror assembly . . . . . . . . . 29

13 Shadow patterns for the sample data . . . . . . . 31

14 Method of finding $n$ corresponding to $\bar{E}$ ' . . . . . 33

15 Method of finding the paraxial shadow magnifications and the distortion coefficients . . . . . . . 34

16. Theoretical and experimental focal properties of the hyperbolic electron mirror . . . . . . . . . 40

B1 Geonetrical relationships for a thick lens . . . . . . 51

B2 Geometrical, relationships of the test apparatus . . . . 51 


\section{INTRODUCTION}

The analogy between electron trajectories and 1 ight rays has been evident since 1828 when Hamilton, in a series of papers published in the Proceedings of the Royal Irish Academy, pointed out the similarities between Fermat's Principle of Least Time and the Principle of Least Action attributed to Maupertuis. The similarities were subsequently experimentally verified by investigators such as Busch [1] who in 1926 shoved that a short, axially symmetric magnetic field could act as a lens for electrons. The theoretical basis for the wave properties of electrons was developed by de Broglie [2] in 1924. The wave properties were verified experimentally by Davisson and Germer [3] in 1927, who observed the diffraction of electrons by crystal surfaces, and almost simultaneously by G. P. Thompson [4], who observed the diffraction of electrons transmitted by thin crystals. Since that time many electron optical devices have been conceived and studied. Among these is the electron mirror. The electron mirror is an electric or magnetic field arranged to reflect incident electrons. As in light optics, a mirror, in addition to reversing the axial component of velocity, can be shaped to perform the function of a converging or diverging lens. The first and the most comprehensive papers in the literature describing electron mirrors are those of Recknagel [5] in 1936, 
Hottenroth [6] in 1937, and $\mathrm{Nicol1} \mathrm{[7]} \mathrm{in} \mathrm{1937.} \mathrm{A} \mathrm{later} \mathrm{work} \mathrm{by}$ Lafferty [8] in 1947 gives some general electron optical properties of mirrors with quadratic elcctrostatic fields. More recently interest in electron mirror microscopy has stimulated further study of electron mirrors. A paper by Mayer [9] in 1959 and two unpublished works by Rempfer [10] and Benedict [1.1] in 1963 describe the electron optical properties of electrostatic mirrors with plane mirror electrodes. The hyperbolic mirror, whose properties are reported in this thesis, is also of the electrostatic type. The focal properties of this mirror can be found analytically and compared with those determined experinentally. This feature, as well as the possible application of the overcorrected spherical aberration which the mirror exhibits in certain ranges of its operation, make this study interesting and useful. 


\section{THEORETYCAL STUDY}

The hyperbolic electron mirror described in this paper has a cylindrically symetric electrostatic field. In the experimental. arrangement, the field is formed between two electrodes as shown in Figure 1. The surface of the negative electrode is a cone of half angle arctan $\sqrt{2}$. The positive electrode is an apertured disk. In the theoretical study of this mirror the equipotential surfaces are assumed to be hyperbolae of revolution except in the immediate vicinity of the positive electrode aperture as shown in Figure 2.

The difference between the flat electrode $A$ used in the experimental study (Figure 1) and the positive electrode of hyperbolic shape used in the theoretical study (Figure 2) is ignored in this thesis for the following reasons: 1) the hyperbola is nearly flat near the axis; 2) the electrons are confined to a region close to the axis in the neighborhood of the electrode A; and 3) the velocity of electrons is high and the effect of the field is relatively small near electrode A.

The aperture, which has the function of allowing electrons to pass into and out of the mirror field, causes the potential to depart from its hyperbolic character locally. The potential distribution is treated as a superposition of the hyperbolic field and a field associated with the aperture. The effects of these two fields on the electron trajectories are considered separately. An electron's 


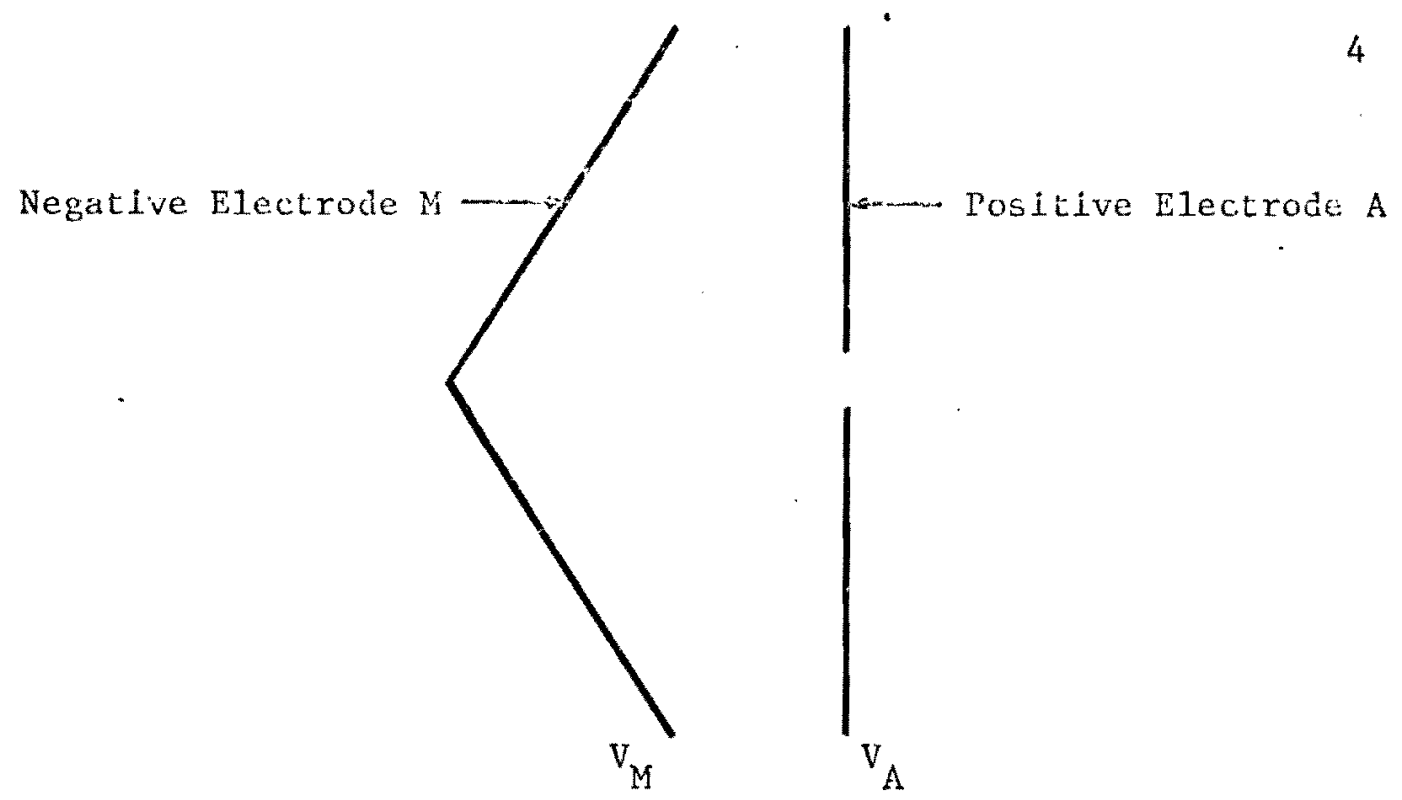

Figure 1. Mirror field used in the experimental study.

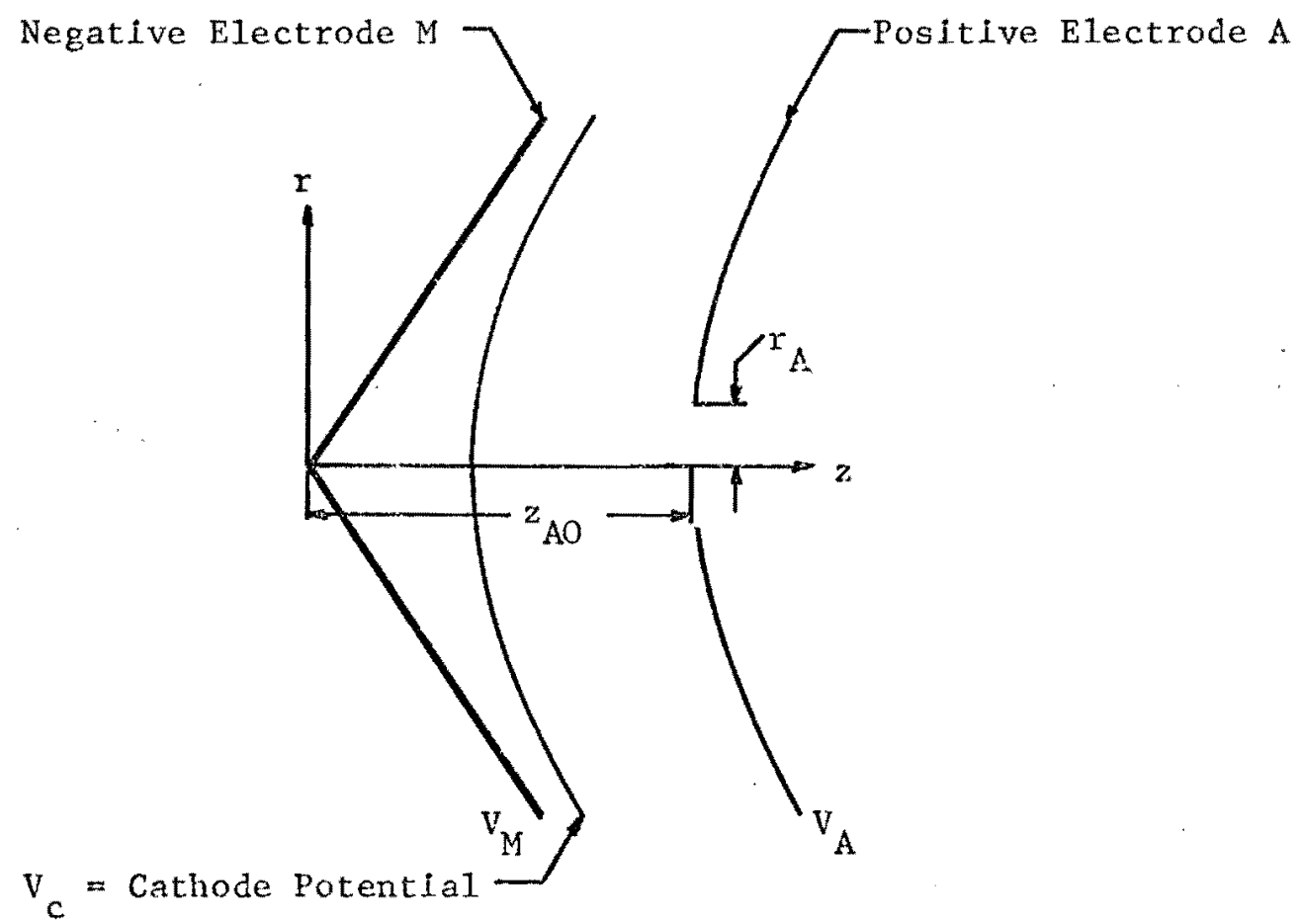

Figure 2. Mirror field used in the theoretical study. 
reflection in the mirror involves first an encounter with the aperture field on entering, second a round trip in the hyperbolic field, and finally another encounter with the aperture field on leaving the mirror. The results obtained here for electron motion apply equali.y well to other charged particles with appropriate polarity of fields since all charged particles entering with the same initial accelerating voltage and jncident path, but differing $q / \mathrm{m}$ ratios, travel along the same trajectory or ray, although not at the same rate. Only the nonrelativistic range is considered.

The electron beam current density is assumed low so that space charge is negligible and the potential $V$ satisfies Laplace's equation,

$$
\frac{\partial^{2} V}{\partial r^{2}}+\frac{1}{r} \frac{\partial V}{r}+\frac{\partial^{2} V}{\partial z^{2}}=0
$$

for the case of rotational symmetry [2].

The separation of variables can be accomplished for the rotationally symetric hyperbolic field by choosing $V=R(r)+Z(z)$. If the $z$ origin is at the vertex of $M$, and $V_{M}$ is taken equal to zero, the solution is

$$
\mathrm{V}=\mathrm{k}\left(\mathrm{z}^{2}-\mathrm{r}^{2} / 2\right)
$$

where, from the boundary conditions in Figure 2, $k=\mathrm{V}_{\mathrm{A}} / \mathrm{z}_{\mathrm{A} 0}{ }^{2}$.

The electric field components $E_{r}$ and $F_{z}$ are functions of only $r$ and $z$ respectively. The motion of electrons in this field can be found by solving Newton's second law for the $r$ and $z$ components separately. For a meridional ray, that is, a ray in a plane containing the optical axis 


$$
\begin{aligned}
& \mathrm{F}_{\mathrm{r}}=\mathrm{m} \ddot{\mathrm{r}}=-\mathrm{eE_{r }}=\mathrm{e} \partial \mathrm{V} / \partial \mathrm{r}=-\mathrm{ekr} \\
& \ddot{\mathrm{r}}+\omega^{2} \mathrm{r}=0 \\
& \mathrm{~F}_{z}=\mathrm{m} \ddot{z}=-e E_{z}=\mathrm{e} \partial \mathrm{V} / \partial z=2 \mathrm{ekz} \\
& \ddot{z}-2 \omega^{2} z=0
\end{aligned}
$$

with $\omega^{2}=\mathrm{ek} / \mathrm{m}$

$$
\text { and } \ddot{x}=d^{2} r / d t^{2} \quad \ddot{z}=d^{2} z / d t^{2} \text {. }
$$

where $e$ is the magnitude of charge and $m$ the mass of the electron.

The solutions of equations ( 3 ) and (4) are parametric equations of motion for electrons in the hyperbolic field as a function of time and are given [13] by

$$
\begin{aligned}
& r=c_{1} \cos \omega t+c_{2} \sin \omega t \\
& z=c_{3} \cosh \sqrt{2} \omega t+c_{4} \sinh \sqrt{2} \omega t
\end{aligned}
$$

For an electron entering the nirror field through the surface $\mathrm{v}_{\mathrm{A}}$ at $\mathrm{t}=0$ with the initial conditions $\mathrm{r}=\mathrm{r}_{\mathrm{A}}, \dot{\mathrm{r}}=\dot{\mathrm{r}}_{\mathrm{A}}, z_{=}=\mathrm{z}_{\mathrm{A}}$, and $\dot{z}=\dot{z}_{A}$, the solutions for $r$ and $z$ become $r=r_{A} \cos \omega t+\dot{r}_{A} / \omega \sin \omega t$ $z=z_{A} \cosh \sqrt{2} \omega t+\left(\dot{z}_{A} / \sqrt{2} \omega\right) \sinh \sqrt{2} \omega t$ These equations can be expressed in the form

$$
\begin{aligned}
& r=\left(r_{A} / \cos \delta\right) \cos \theta \\
& z=\left(z_{A} / \cosh \varepsilon\right) \cosh \phi
\end{aligned}
$$

with $\theta=\omega t-\delta$ 
$\phi=\sqrt{2} \omega t-\varepsilon$

$\tan \delta=\dot{\mathrm{r}}_{\mathrm{A}} / \omega \mathrm{r}_{\mathrm{A}}$

$\tanh \varepsilon=-\dot{\mathrm{z}}_{\mathrm{A}} / \sqrt{2} \omega \mathrm{z}$

The values of $r, z, \dot{r}, \dot{z}$ for the electron leaving the hyperbolic field are given by

$$
\begin{aligned}
& r_{A}^{\prime}=\left(r_{A} / \cos \delta\right) \cos \theta^{\prime} \\
& { }_{z_{A}}^{\prime}=\left(z_{A} / \cosh \varepsilon\right) \cosh \phi^{\prime} \\
& \dot{x}_{A}^{\prime}=-\omega r_{A}^{\prime} \tan \theta^{\prime} \\
& \dot{z}_{A}^{\prime}=\sqrt{2} \omega z_{A}^{\prime} \tanh \phi^{\prime}
\end{aligned}
$$

where $\theta^{\prime}=\omega t^{\prime}-\delta$

$$
\phi^{\prime}=\sqrt{2} \omega t^{\prime}-\varepsilon
$$

and $t^{\prime}$ is the time which the electron spends in the mirror field.

In the following steps the time $t^{\prime}$ is evaluated. Expressions (13) through (18) are expanded in terms of $r_{A}$ and $\dot{r}_{A}$, and terms above second order are omitted. In the paraxial approximation (where the distance from the axis and the slope $\dot{\mathrm{r}}_{\mathrm{A}} / \dot{\mathrm{z}}_{\mathrm{A}}$ remain sma11) the second order terms are neglected. From (2)

$$
k\left(z_{A}^{2}-r_{A}^{2 / 2)}=k z_{A O}^{2}\right.
$$

or $z_{A}^{2}=z_{A 0}^{2}+r_{A}^{2 / 2}=z_{A O}^{2}\left(1+2 \sigma^{2}\right)$

where $\sigma=\mathrm{r}_{\mathrm{A}} / 2 \mathrm{z}_{\mathrm{AO}}$

By the binominal expansion

$$
\mathrm{z}_{\mathrm{A}}=\mathrm{z}_{\mathrm{AO}}\left(1+\sigma^{2}\right)
$$


Likewise for the exiting ray

$$
z_{\Lambda}^{\prime}=z_{A O}\left(1+\sigma^{\prime 2}\right)
$$

where $\sigma^{\prime}=\mathrm{r}_{\mathrm{AO}}{ }^{\prime / 2} \mathrm{z}_{\mathrm{AO}}$

The electron speed $v_{A}$ at $V_{\Lambda}$ is found from

$$
v_{A}^{2}=\left(-\dot{z}_{A}\right)^{2}+\left(\dot{r}_{A}\right)^{2}=2(e / m)\left(V_{A}-v_{C}\right)=2 \gamma^{2} \omega^{2} z_{A O}{ }^{2}
$$

where $\gamma^{2}=\left(V_{A}-V_{C}\right) /\left(V_{A} \cdots V_{M}\right)$ is the ratio of the accelerating voltage to the mirror voltage as shown in Figure 2. Then

$$
-\dot{\mathrm{z}}_{\mathrm{A}}=\mathrm{v}_{\mathrm{A}} /\left(1+\dot{\mathrm{r}}_{\mathrm{A}}{ }^{2 / \dot{\mathrm{z}}_{\mathrm{A}}}{ }^{2}\right)^{1 / 2}=\sqrt{2} \gamma_{\mathrm{AO}}\left(1-\dot{\mathrm{r}}_{\mathrm{AO}}{ }^{2 / 2} \dot{\mathrm{z}}_{\mathrm{AO}}{ }^{2}\right)
$$

The paraxial values (with subscript zero) of $z_{A}, z_{A}^{\prime}$, and $\dot{z}_{\Lambda}$ are

$$
\begin{aligned}
& \left(z_{A}\right)_{0}=z_{A O} \\
& \left(z_{A}^{\prime}\right)_{O}=z_{A O} \\
& \left(-\dot{z}_{A}\right)_{O}=v_{A}=\sqrt{2} \gamma \omega z_{A O}
\end{aligned}
$$

Substitution of the paraxial values of these quantities into equation (12) gives

$$
\tanh \varepsilon_{0}=\gamma
$$

from which

$$
\cosh \varepsilon_{0}=\frac{1}{\sqrt{1-r^{2}}}
$$

From these values and equation (14)

$$
\cosh \phi_{0}^{\prime}=\cosh \varepsilon_{0}
$$

or

$$
\phi_{0}^{\prime}=\varepsilon_{0}=\operatorname{arctanh} \gamma
$$


Then from equation (18) the tine which the paraxial electrons spend In the mirror field is given by

$$
\omega t_{0}^{\prime}=\left(\phi_{0}^{\prime}+\epsilon_{0}\right) / \sqrt{2}=\sqrt{2} \operatorname{arctanh} \gamma
$$

Substituting the value for the time into the solutions (13), (15), and (16) for the exiting ray gives, in paraxial form,

$$
\begin{aligned}
& r_{A O}{ }^{\prime}=r_{A} \cos \left(\omega t_{O}{ }^{\prime}-\delta_{O}\right) / \cos \delta_{O} \\
& \dot{r}_{A O}{ }^{\prime}=-\omega r_{A O} \cdot \tan \left(\omega t_{O}{ }^{\prime}-\delta_{O}\right) \\
& \dot{z}_{A O}{ }^{\prime}=\sqrt{2} \omega z_{A O} \gamma=v_{A}
\end{aligned}
$$

The quantity $\delta$ involves the initial conditions $\dot{\mathrm{r}}_{\mathrm{A}} / \mathrm{r}_{\mathrm{A}}$ with which an electron begins its motion in the hyperbolic field. This ratio depends on both the path of the incident electron outside of the mirror and the effect of the aperture field. If the electron trajectory is initially parallel to the z-axis, the radial velocity component with which the electron enters the hyperbolic field is due only to the field around the aperture in $A$. The properties of aperture fields of this type vere first reported by Davisson and Calbick [14]. For sma11 apertures the field has the effect of a thin lens of focal length

$$
f=\frac{4 V_{B}}{(\partial V / \partial z)_{2}-(\partial V / \partial z)_{1}}
$$

where $V_{B}$ is the bearn voltage at the aperture, defined by $1 / 2 \mathrm{mv}^{2}$ $=\mathrm{eV}_{\mathrm{B}}$, and the derivatives in the denominator are the gradients of the potential on the two sides of the electrode which would have existed in the absence of the aperture. In the present case, with 
$\mathrm{v}_{\mathrm{M}}=0$,

$$
\begin{aligned}
& V_{B}=V_{A}-V_{C}=\gamma^{2}\left(V_{A}-V_{M}\right)=\gamma^{2} V_{A} \\
& (\partial V / \partial z)_{1}=2 k z_{A} \\
& (\partial V / \partial z)_{2}=0
\end{aligned}
$$

and the focal length of the aperture lens is

$$
E_{A}=\frac{4 \gamma^{2} V_{A}}{-2 k{ }_{A}}=\frac{-2 \gamma^{2} z_{A O}{ }^{2}}{z_{A}}
$$

The paraxial focal length is

$$
f_{A O}=-2 \gamma^{2} z_{A O}
$$

From Figure (3)

$$
\frac{\dot{\mathrm{r}}_{\mathrm{A}}}{\mathrm{r}_{\mathrm{A}}}=\frac{\dot{\mathrm{z}}_{\mathrm{A}}}{\mathrm{f}_{\mathrm{A}}}
$$

then from (11)

$$
\tan \delta=\dot{r}_{A} / \omega r_{A}=\dot{z}_{A} / \omega f_{A}=\frac{-\dot{z}_{A}{ }_{A}}{2 \omega \gamma^{2} z_{A O}{ }^{2}}
$$

and

$$
\tan \delta_{0}=\frac{\dot{z}_{A O}}{\omega f_{A O}}=\frac{\sqrt{2} \gamma \omega z}{\omega 2 \gamma^{2} z_{A O}}=\frac{1}{\sqrt{2} \gamma}
$$

From (9), (28), and (38), the paraxial value of $0^{\prime}$ is

$$
\theta_{0}^{\prime}=\omega t_{0}^{\prime}-\delta_{0}=\sqrt{2} \operatorname{arctanh} \gamma-\arctan \frac{1}{\sqrt{2} \gamma}
$$

The ratio $-\dot{\mathbf{r}}_{\mathrm{A}}{ }^{\prime} / \dot{z}_{\mathrm{A}}$ ' gives the slope of the trajectory as the electron leaves the hyperbolic field, and is the tangent of the angle $\beta^{\prime}$ at which the electron is incident on the aperture lens 


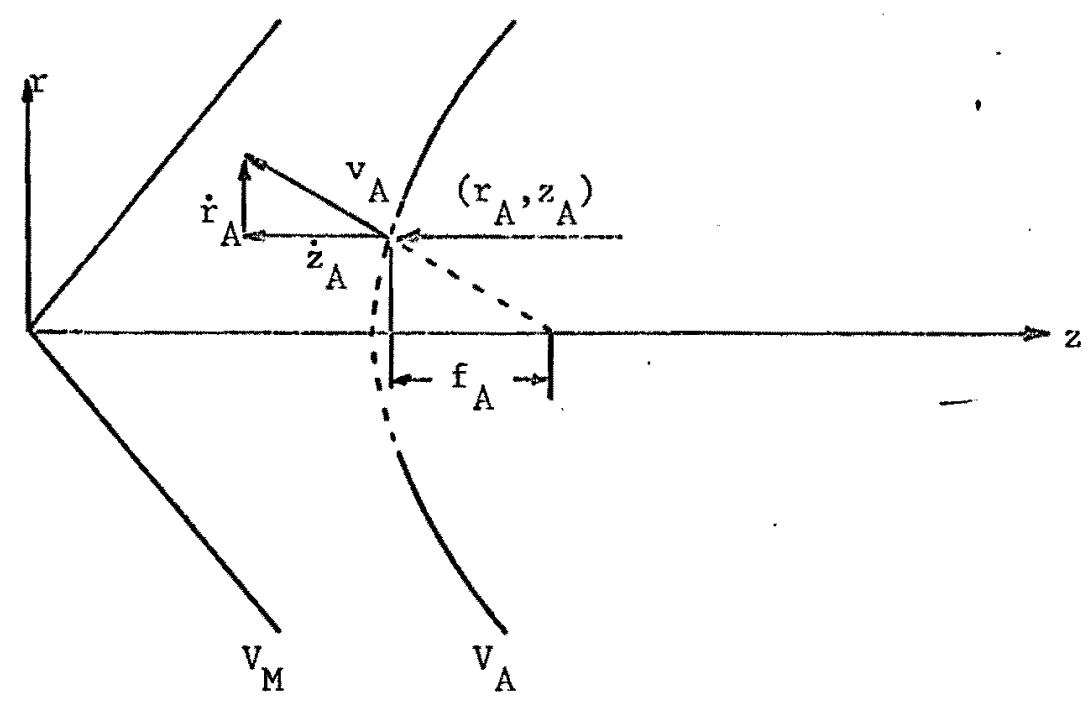

Figure 3. Deflection of an incident ray by the aperture lens.

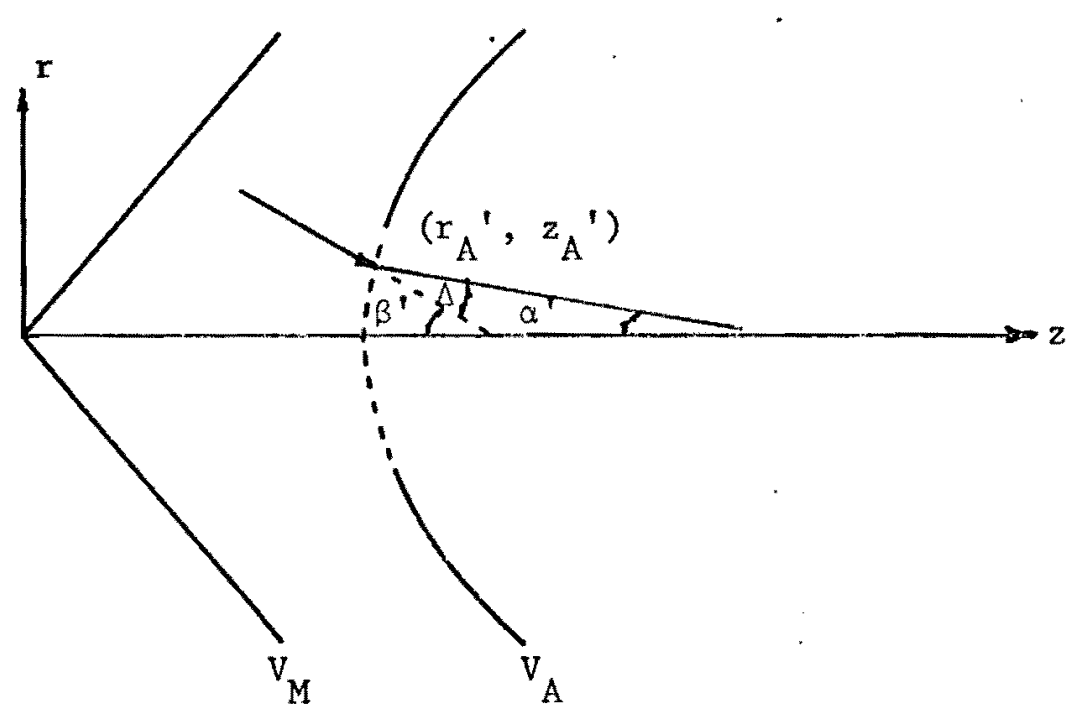

Figure 4. Deflection of a reflected ray by the aperture lens. 
on its way out as shown in Figure 4. From (15) and (16)

$$
\tan \beta^{\prime}=-\dot{r}_{A^{\prime}} / \dot{z}_{A}^{\prime}=\frac{r_{A}^{\prime}}{\sqrt{2} z_{A}} \cdot \frac{\tan \theta^{\prime}}{\tanh \phi^{\prime}}
$$

The paraxial value of $\beta^{\prime}$ is given by

$$
\tan \beta_{0}^{\prime}=-\dot{r}_{A O}{ }^{\prime} / \dot{z}_{A O}{ }^{\prime}=\frac{{ }_{A O}{ }^{\prime}}{\sqrt{2} \gamma^{2}{ }_{A O}} \tan \theta_{0}^{\prime}
$$

The emerging ray is deflected by the aperture lens through an angle given by

$$
\tan \Delta^{\prime}=-r_{A}^{\prime} / f_{A}^{\prime}=\frac{r_{A}^{\prime} z_{A}^{\prime}}{2 \gamma^{2} z_{A O}{ }^{2}}
$$

where $\mathrm{f}_{\mathrm{A}}{ }^{\prime}=-2 \gamma^{2} \mathrm{z}_{\mathrm{AO}}{ }^{2 / \mathrm{z}_{\mathrm{A}}}{ }^{\prime}$

and $\mathrm{f}_{\mathrm{AO}}{ }^{\prime}=-2 \gamma^{2} \mathrm{z}_{\mathrm{AO}}$

The paraxial value of $\Delta^{\prime}$ is given by

$$
\tan \Delta_{0}^{\prime}=r_{A O}{ }^{\prime} /-f_{A O}{ }^{\prime}=r_{A O}{ }^{\prime} / 2 \gamma^{2} z_{A O}=\frac{r_{A O}{ }^{\prime}}{\sqrt{2} \gamma z_{A O}} \cdot \tan \delta_{O}
$$

The angle $\alpha^{\prime}=\beta^{\prime}-\Delta^{\prime}$ at which the ray leaves the mirror, after diverging action by the aperture lens, is found from the trigonometric relationship

$$
\tan \alpha^{\prime}=\frac{\tan \beta^{\prime}-\tan \Delta^{\prime}}{1+\tan \beta^{\prime}}
$$

The paraxial value of $\alpha^{\prime}$ is given by

$$
\begin{aligned}
\tan \alpha_{0}^{\prime} & =\tan \beta_{0}^{\prime}-\tan \Delta_{0} \\
& =\frac{r_{A O}^{\prime}}{\sqrt{2} \gamma{ }_{A O}}\left(\tan \theta_{0}^{\prime} \cdots \tan \delta_{0}\right)
\end{aligned}
$$




$$
=\frac{{ }_{A}}{\sqrt{2} \gamma{ }^{z}{ }_{A O}} \frac{\cos \theta_{0}^{\prime}}{\cos \delta_{0}}\left(\tan \theta_{0}^{\prime}-\tan \delta_{0}\right)
$$

From Figure 5 the focal length of the hyperbolic electron

mirror is

$$
f=r_{A} / \tan \alpha^{\prime}
$$

and the paraxial focal length is

$$
f_{0}=r_{A} / \tan \alpha_{0}^{\prime}=\sqrt{2} \gamma z_{A O} \frac{\cos \delta_{0}}{\cos \theta_{0}^{\prime}} \frac{1}{\left(\tan \theta_{0}^{\prime}-\tan \delta_{0}\right)}
$$

From Figure 5 the focal distance is

$$
g=z_{A}^{\prime}+r_{A}^{\prime} / \tan \alpha^{\prime}
$$

and the paraxial focal distance is

$$
g_{0}=z_{A O}\left[1+(\sqrt{2} \gamma) /\left(\tan \theta_{0}^{\prime}-\tan \delta_{0}\right)\right]
$$

where $\delta_{0}$ and $\theta_{0}^{\prime}$, as seen from (38) and (39), are functions of $\gamma$ alone. The paraxial focal properties, $\mathrm{f}_{0}$ and $\mathrm{g}_{0}$, thus depend only on $\gamma$ and $z_{A O}$.

The focal length and focal distance are found to second order in $\mathrm{r}_{\mathrm{A}}$ by including the second order terms in the expressions derived in this section. These second order calculations are carried through in Appendix A, and expressions for the aberration coefficients are given there.

The results of the theoretical study are expressed in terms of the paraxial values of $f_{0}$ and $g_{0}$ and the second order spherical aberration coefficients $\mathrm{C}_{\mathrm{f}}$ and $\mathrm{C}_{\mathrm{g}}$ of these quantities defined by 


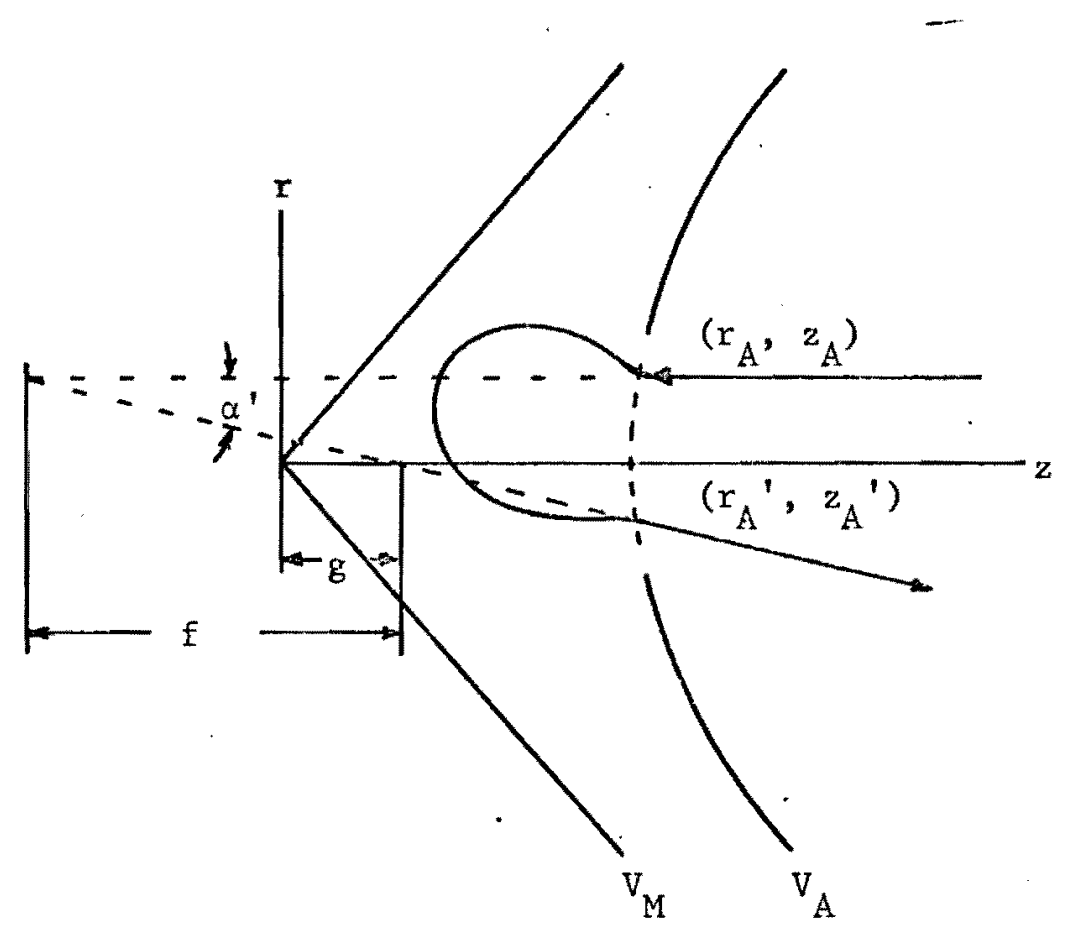

Figure 5. Focal length and focal distance of the mirror. 


$$
\frac{-\Delta f}{f_{0}}=C_{f}\left(\frac{\rho}{f_{0}}\right)^{2}
$$

and

$$
\frac{-\Delta g}{f_{0}}=C_{g}\left(\frac{\rho}{f_{0}}\right)^{2}
$$

where $\Delta f$ and $\Delta g$ are the variations of focal length and distance with the height $p$ of the incident ray on the mirror. In this study $p=r_{A}$. The theoretical values of $\mathrm{f}_{0}, \mathrm{~g}_{0}, \mathrm{C}_{\mathrm{f}}, \mathrm{C}_{\mathrm{g}}$, are given in Table $\mathrm{I}$ for values of $\gamma^{2}$ between .99 and .92 . 
TABLE I

THEORETICAL RESULTS

\begin{tabular}{|c|c|c|c|c|}
\hline$\gamma^{2}$ & $f_{0}$ & $g_{0}$ & $C_{f}$ & $C_{g}$ \\
\hline .990 & 2.205 & -2.068 & $\alpha^{\infty}$ & -6515 \\
\hline .980 & .524 & -.305 & 87.2 & -168 \\
\hline .971 & .394 & -.126 & 19.4 & -31.9 \\
\hline .962 & .345 & -.037 & 7.3 & -16.6 \\
\hline .943 & .308 & .070 & 1.3 & -8.1 \\
\hline .926 & .300 & .132 & -.1 & -6.0 \\
\hline
\end{tabular}




\section{EXPERTMENTAL STUDY}

The study made to experimentally determine the focal properties of the hyperbolic electron mirror is presented in this section.

Method

The focal properties of the electron mirror are determined by a ray tracing method of Spangenberg and Field [15] extended by Rempfer [16] to include the aberrations of focal length and position. The method, adapted for use with a mirror, consists of the analysis of the shadow pattern formed by a fine grating of parallel wires placed in the electron beam as shown in Figure 6 .

Electrons from a "point" source pass through a sma11 opening in the viewing screen and illuminate the mirror. The electron beam is reflected by the mirror and falls on the viewing screen. The grating casts shadows in both the incident and reflected beams. Outside the mirror the electrons are in the field free space and the paths are straight, making it possible to trace the rays in object and image space. The positions of the mirror electrodes used in the experimental study are indicated in the figure.

The description of this method of ray tracing is facilitated if the optical system is drawn as a transmission system so that incident and reflected rays do not overlap. Figure 7 shows the 


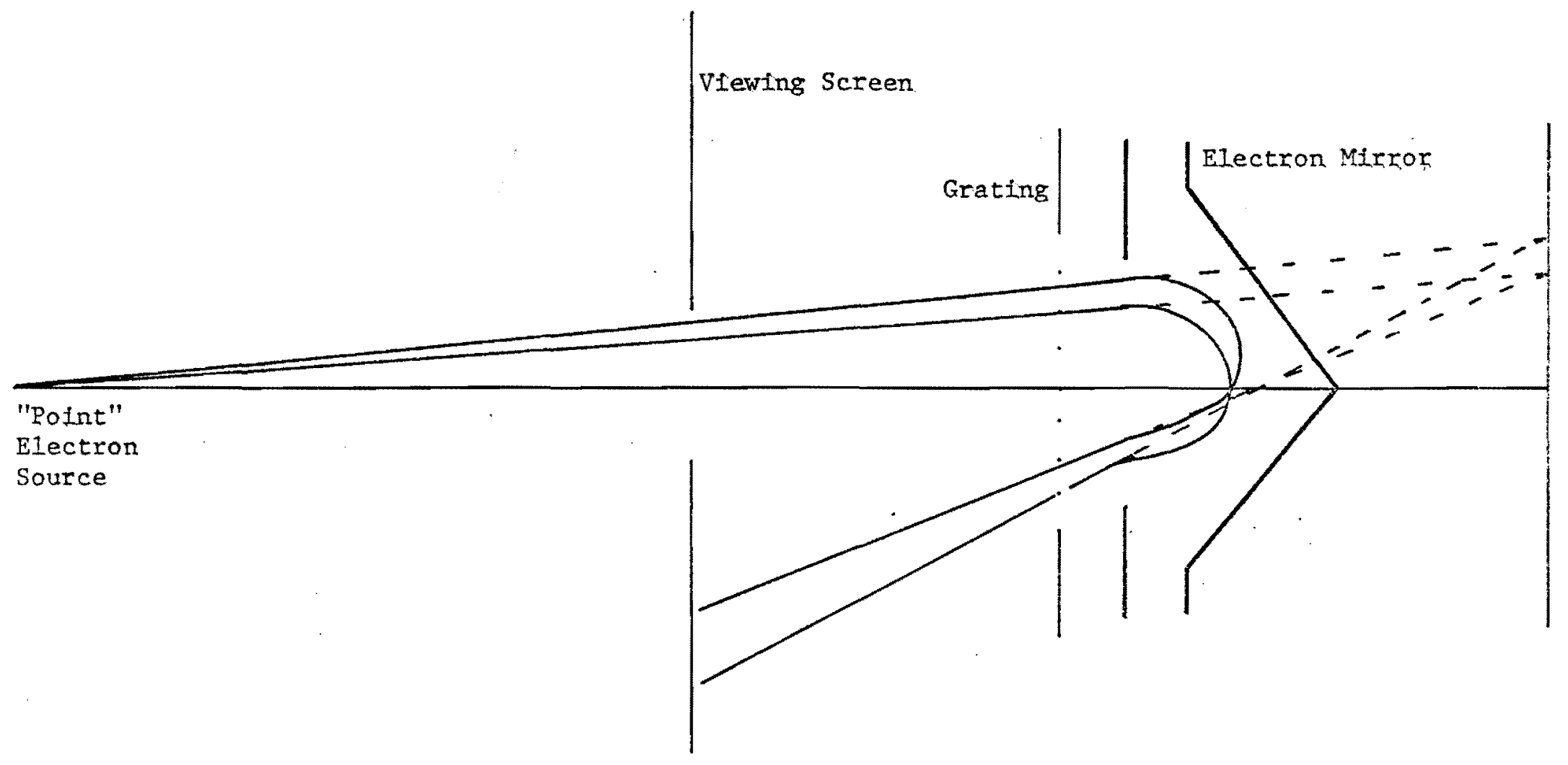

Figure 6. Experimental Mirror Arrangement 


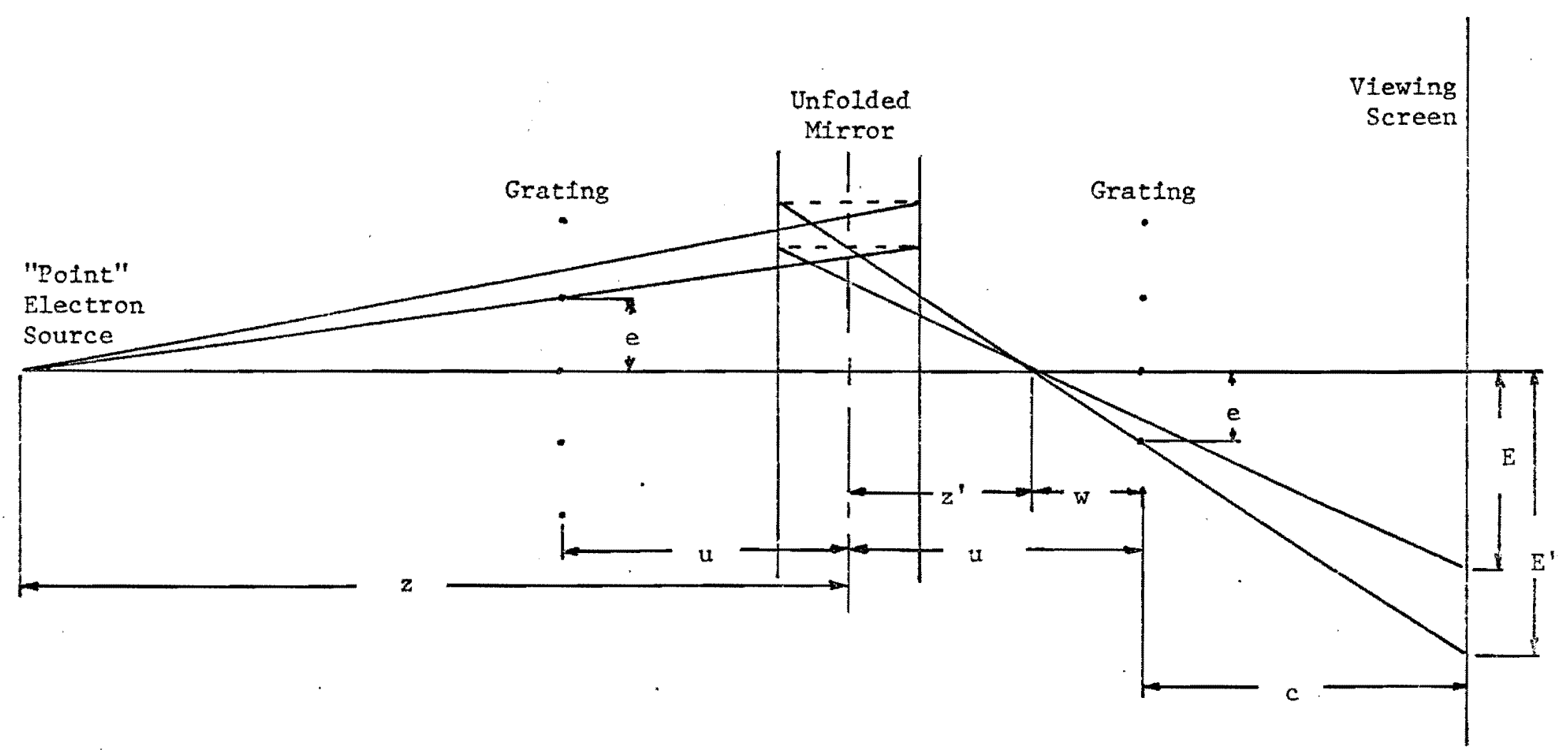

Figure 7. Equivalent optical system and ray tracing method. 
equivalent optical system unfolded about $z=0$, the vertex of the mirror electrode. The effects of aberrations have been ignored in both Figures 6 and 7 .

With the vertex of the cone-shaped electrode as the origin, the focal length $f$ and the focal. distance $g$ are given (from Appendix $B$ ) in terns of the source distance $z$, image distance $z^{\prime}$ and lateral magnification $\mathrm{m}$ by

$$
\begin{aligned}
& f=\frac{z-z^{\prime}}{1 / m-m} \\
& g=z^{\prime}-f_{i n}
\end{aligned}
$$

The quantities $z^{\prime}$ and $m$ are expressed in terms of the distances $u$ and $c$ in Figure 7 by

$$
\begin{aligned}
& u-z^{\prime}=\frac{c}{M^{\prime}-1} \\
& m=\frac{M^{\prime}}{M} \frac{1}{M^{\prime}-1} \frac{c}{z-u}
\end{aligned}
$$

where $M$ and $M$ ' are the magnifications of the grating shadows formed in the incident and reflected beams respectively.

In the case where the electron source is distant and the fncident rays are parallel, the expressions for focal length and focal distance simplify to

$$
\begin{aligned}
& f=\frac{c}{M\left(1-1 / M^{\prime}\right)} \\
& g=z^{\prime}=u-\frac{c}{M^{\prime} \cdot 1}
\end{aligned}
$$

Spherical aberration is the variation of focal length and focal distance with height of the incident rays. This aberration, which 
causes rays incident at different heights to cross the axis at dif-ferent positions and also causes the principle planes to be curved, results in distortions of the grating shadows. The distortions of the grating shadovs can be used to calculate the aberrations. For the usual undercorrected focal point aberration, the focal distance is smaller for rays incident farther from the axis. This causes the grating, placed as it is between the image of the source and the screen, to be projected with barrel distortion by the emerging beam. For overcorrected focal point aberration, the focal distance increases with the height of the ray and causes the grating shadow in the emerging beam to be projected with pincushion distortion. The effect of undercorrected focal point aberration on the shadow magnification is shown in Figure 8 for the case of incident rays parallel to the axis.

In the case of focal length aberration, as with focal point aberration, undercorrection corresponds to a decrease in focal length, and overcorrection to an increase in focal length, with height of incidence. This aberration manifests itself mainly in the shadow cast by the grating in the incident beam, which exhibits pincushion distortion for undercorrection and barrel distortion for overcorrection. The effect of undercorrected focal length aberration is also shown in Figure 8.

The grating distortions are approximately parabolic under the experimental conditions used, and the shadow magnifications can be 


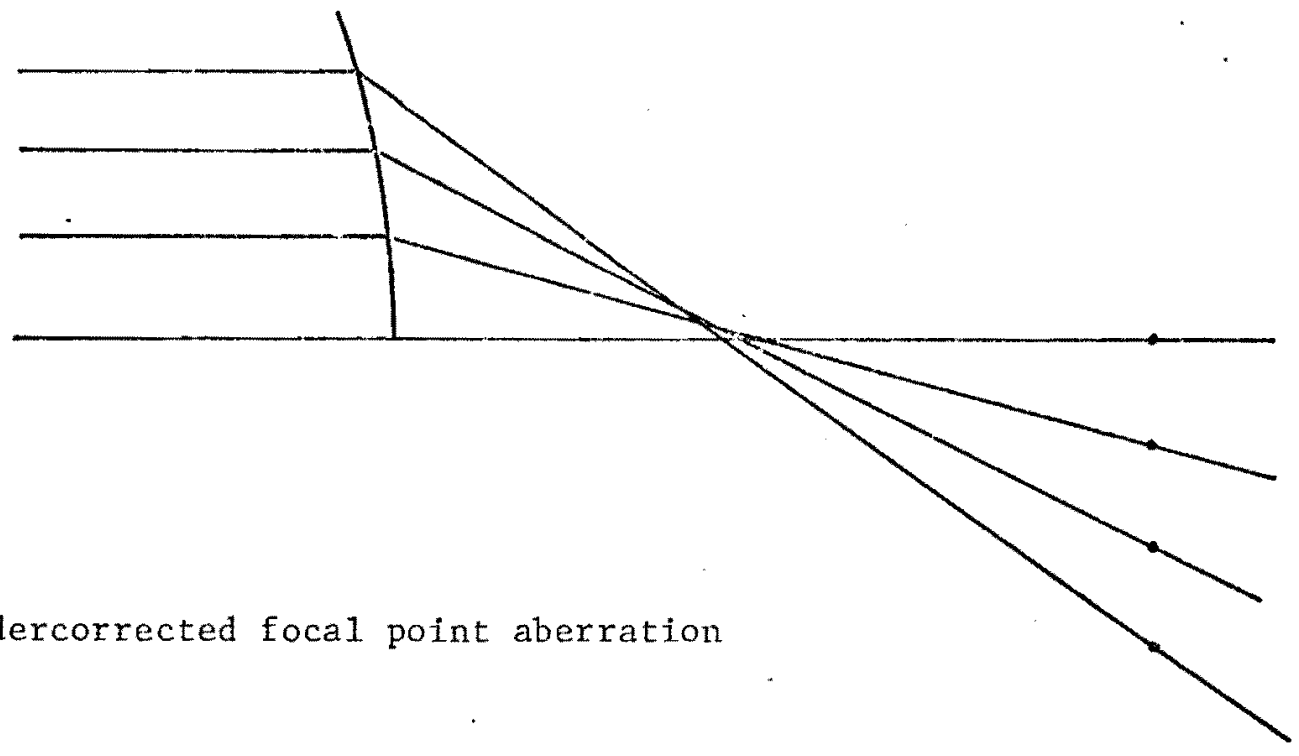

Undercorrected focal point aberration

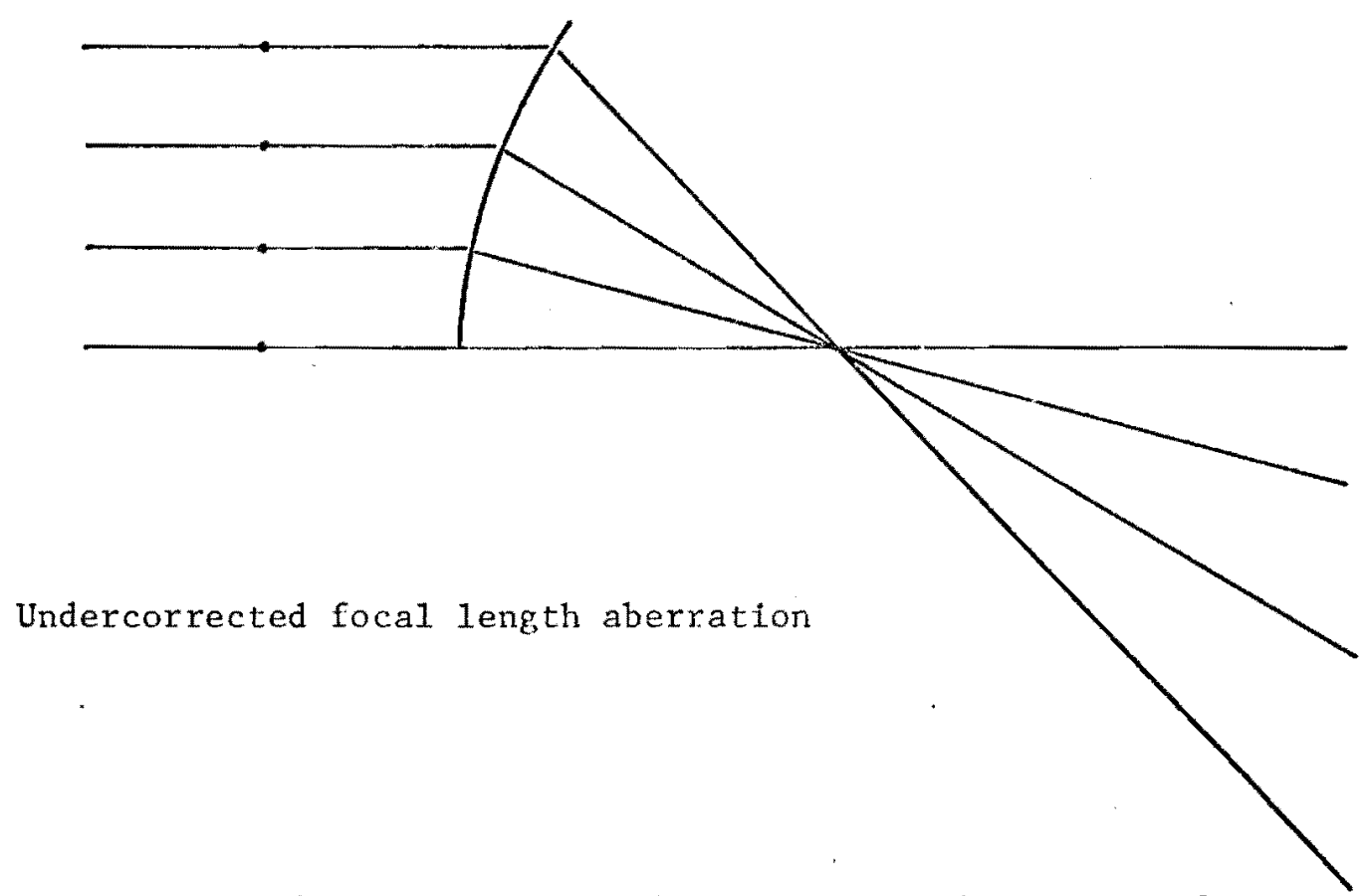

Figure 8. Undercorrected geometrical aberrations for parallel incidence. 
expressed with sufficient accuracy as

$$
\begin{aligned}
& M=M_{0}\left(1+\beta n^{2}\right) \\
& M^{\prime}=M_{0}^{\prime}\left(1+\beta^{\prime} n^{2}\right)
\end{aligned}
$$

where $M_{0}$ and $M_{0}$ ' are the paraxial magnifications, $\beta$ and $\beta^{\prime}$ are the distortion coefficients, and $\mathrm{n}$ is the distance off axis of the incident ray in terms of grating spacings.

When the expressions for the shadow magnifications (60) and (61) are substituted into equations (54) and (55) the expressions

- for focal length and focal distance can be separated into paraxial and aberration terms

$$
\begin{aligned}
& f=f_{0}-C_{f} f_{0}\left(\rho / f_{0}\right)^{2} \\
& g=g_{0}-C_{g} f_{0}\left(\rho / f_{0}\right)^{2}
\end{aligned}
$$

where $f_{0}=\frac{z-z_{0}{ }^{\prime}}{1 / m_{0}-m_{0}}$

$$
\begin{aligned}
& \mathrm{g}_{0}=\mathrm{z}_{0}^{\prime}-\mathrm{f}_{0} \mathrm{~m}_{0} \\
& \mathrm{u}-\mathrm{z}_{0}^{\prime}=\frac{\mathrm{c}}{\mathrm{M}_{0}^{\prime}-1} \\
& \mathrm{~m}_{0}=\frac{\mathrm{M}_{0}^{\prime}}{\mathrm{M}_{0}} \frac{\mathrm{u}-\mathrm{z}_{0}^{\prime}}{\mathrm{z}-\mathrm{u}}
\end{aligned}
$$

The aberration coefficients $\mathrm{C}_{\mathrm{f}}$ and $\mathrm{C}_{\mathrm{g}}$ are derived in Appendix $\mathrm{B}$ and are given by

$$
c_{f}=\frac{1}{\left[\frac{1}{m_{0}}+1\right]^{2}\left[\frac{e}{z-u}\right]^{2}}\left[\frac{1+m_{0}^{2}}{1-m_{0}^{2}} a-m_{0} b\right]
$$




$$
\begin{aligned}
& C_{g}=\frac{1}{\left[\frac{1}{m_{0}}+1\right]^{2}\left[\frac{e}{z-u}\right]^{2}}\left[\frac{-2 m_{0}}{1-m_{0}{ }^{2}} a-b\right] \\
& \text { with } \left.a=\beta+\frac{\beta^{\prime}}{M_{0}^{\prime}-1}\right] \\
& b=\frac{z-u}{z-z_{0}^{\prime}} \frac{M_{0}}{M_{0}^{\prime}-1} \beta^{\prime}
\end{aligned}
$$

where $e$ is the grating spacing and $\rho$ is the height on the first principle plane of the incident ray.

\section{Apparatus and Procedure}

A photograph of the electron mirror assembly is shown in Figure 9. The mirror field is formed between two machined brass electrodes. The positive electrode is a disk with an axial aperture. The negative electrode is a cone of half-angle arctan $\sqrt{2}$. The regative electrode is held in position by insulating Rexolite spacers and axially aligned along with the positive electrode and the grating used for ray tracing in a brass mounting cell. The grating is shown in the foreground of Figure 9. The film holder, also shown in Figure 9, has a shallow cavity machined in a brass housing and a hinged lid with a phosphor viewing screen deposited on the outside. The holder has an axial aperture allowing electrons to pass from the source to the mirror.

The electron source consists of a thoriated tungsten hairpin filament electron gun, a condenser lens, and a pinhole stop. The 


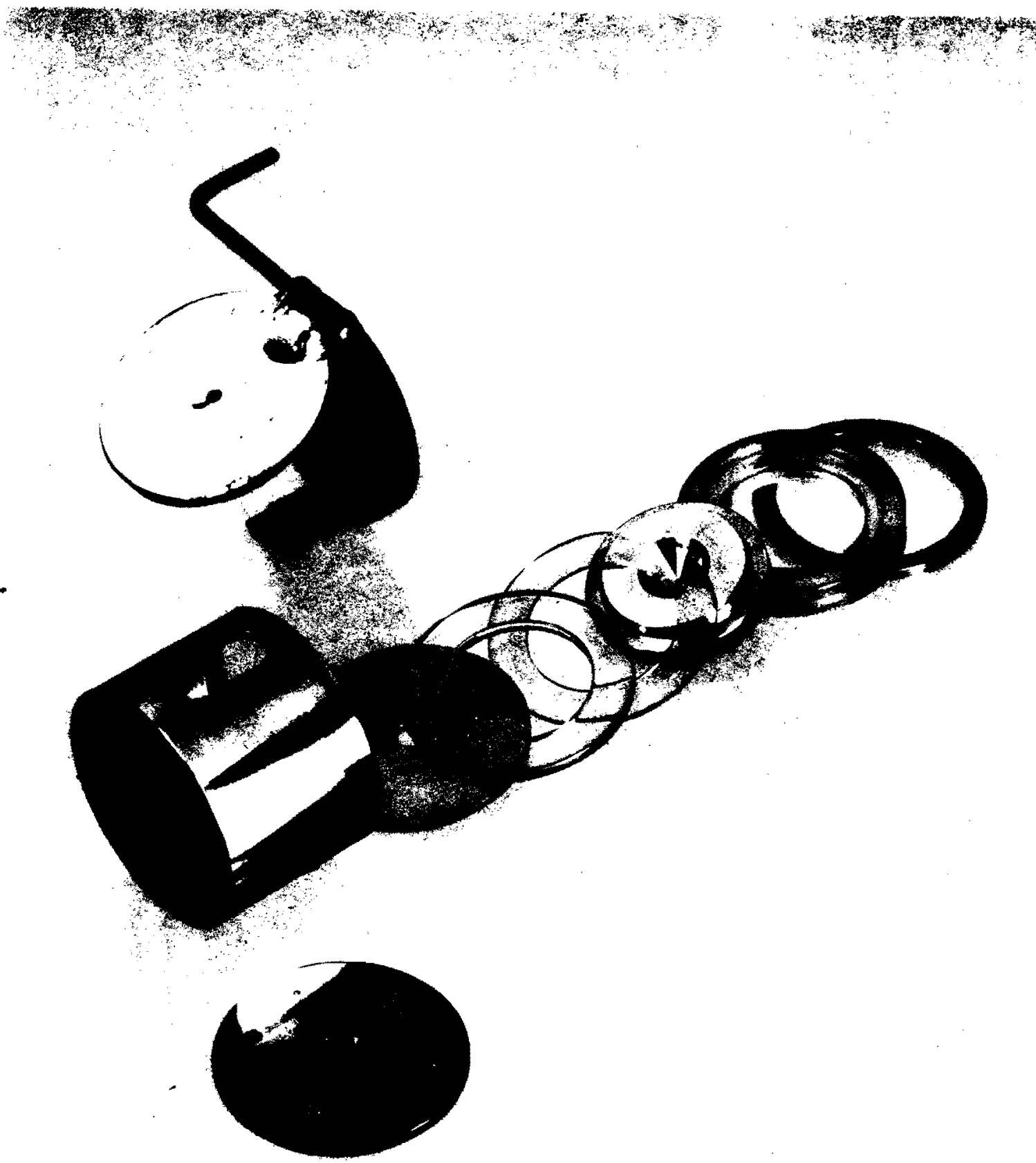

Figure 9. Photograph of film holder, mirror assembly and grating. 
electron source is aligncd with the film holder and mirror cell in the $\mathrm{V}$-ways of an electron optical bench as shown in Figure 10. The optical bench is mounted on a Veeco pumping station.

The electron optical properties of the mirror were experimentally investigated at each of six voltage ratios $\gamma^{2}=\left(\mathrm{V}_{\mathrm{A}}-\mathrm{V}_{\mathrm{C}}\right)$ / $\left(V_{A}-V_{M}\right)$ where $V_{A}$ is the apertured electrode potential and $V_{M}$ is the mirror electrode potential. In the experimental case it was convenient to have $V_{A}=0$. The cathode potential $V_{C}$ was $-20 \mathrm{kV}$ in al1 cases. The mirror potential $\mathrm{V}_{\mathrm{M}}$ was obtained by adding to the cathode potential the voltage across one or more 200 volt zener diodes. A diagram of the electrical circuit is shown in Figure 11. The tolerance of the zener diodes was $\pm 5 \%$ and the value of $\gamma^{2}$ was known to better than $\pm .1 \%$. For each voltage ratio, film cut as an apertured disk was placed in the film holder. The optical bench was evacuated to about $5 \times 10^{-5}$ torr. The electron source and mirror voltages were applied and the electron source adjusted to give suitable illumination of the pattern on the phosphor screen. The film holder lid was raised by means of a mechanical feed-through in the sjde of the optical bench. The film was exposed from ten to twenty seconds depending upon the electron beam intensity. The voltages were disconnected and the bench vented to complete one exposure cycle. The positions of the grating shadows on the developed film were measured with a traveling microscope.

A dimensioned drawing of the mirror assembly is shown in Figure 12. The distance between the grating and mirror electrode 


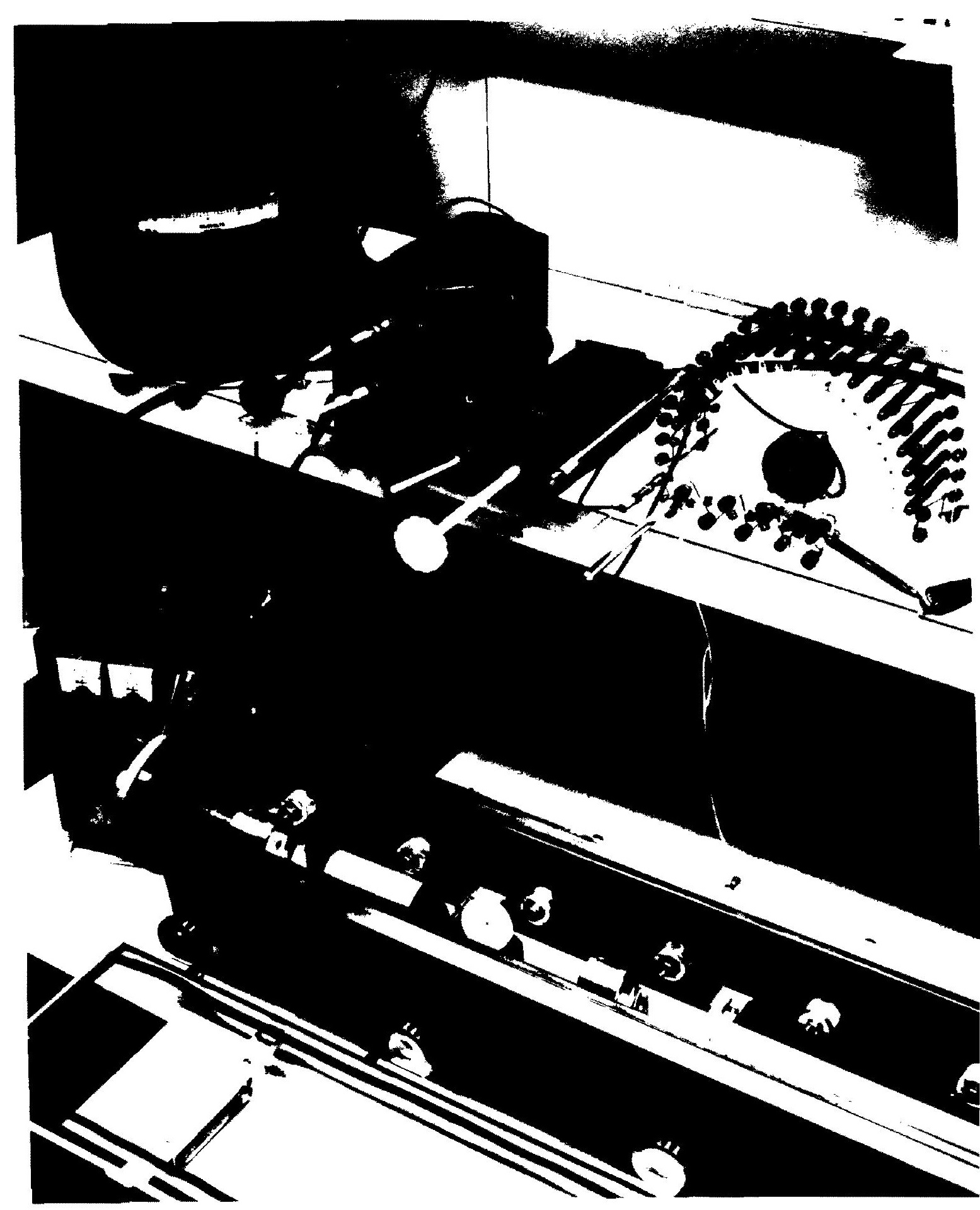

Figure 10. Photograph of experimental set-up. 


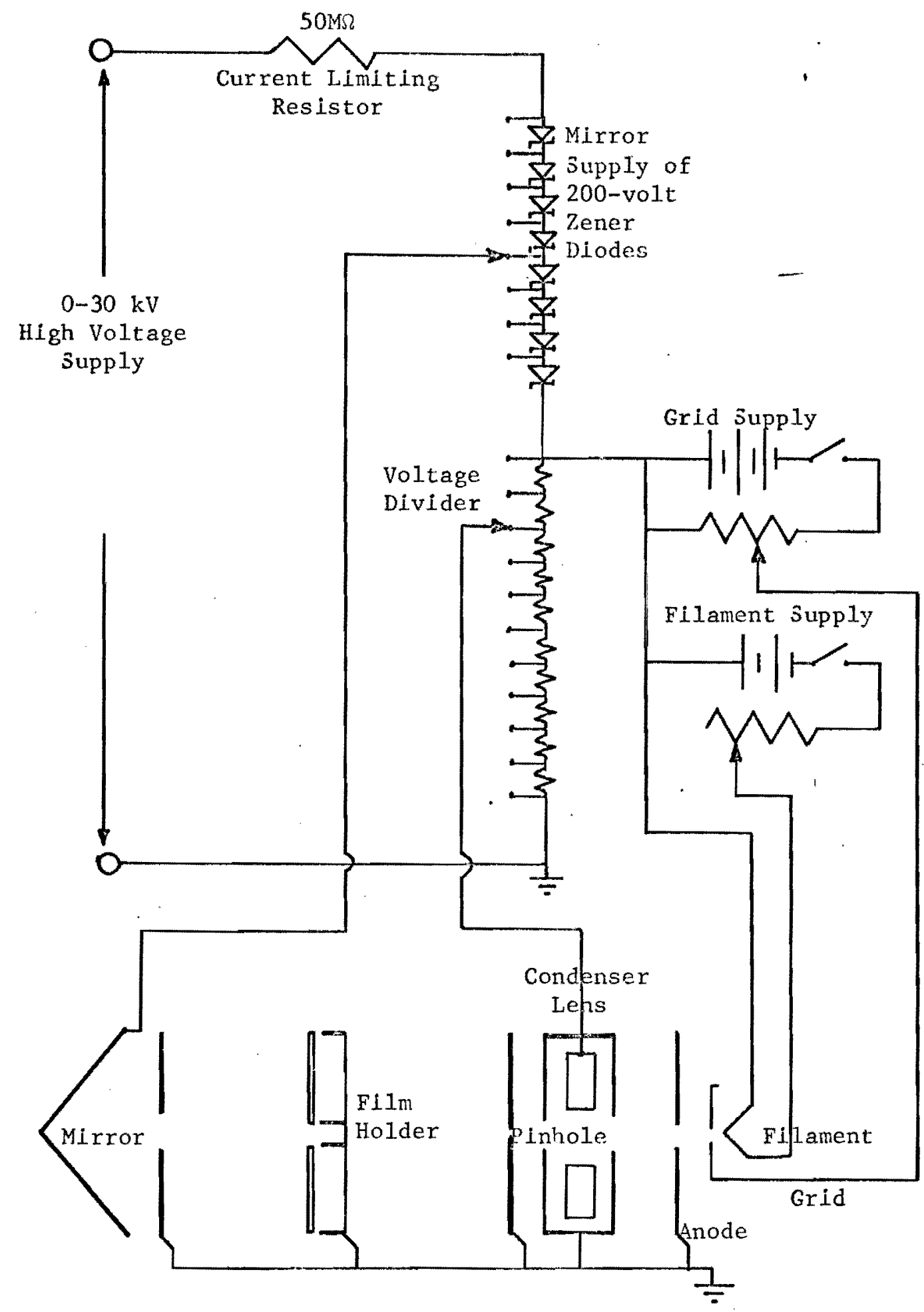

Eigure 11. Electrical circuit. 


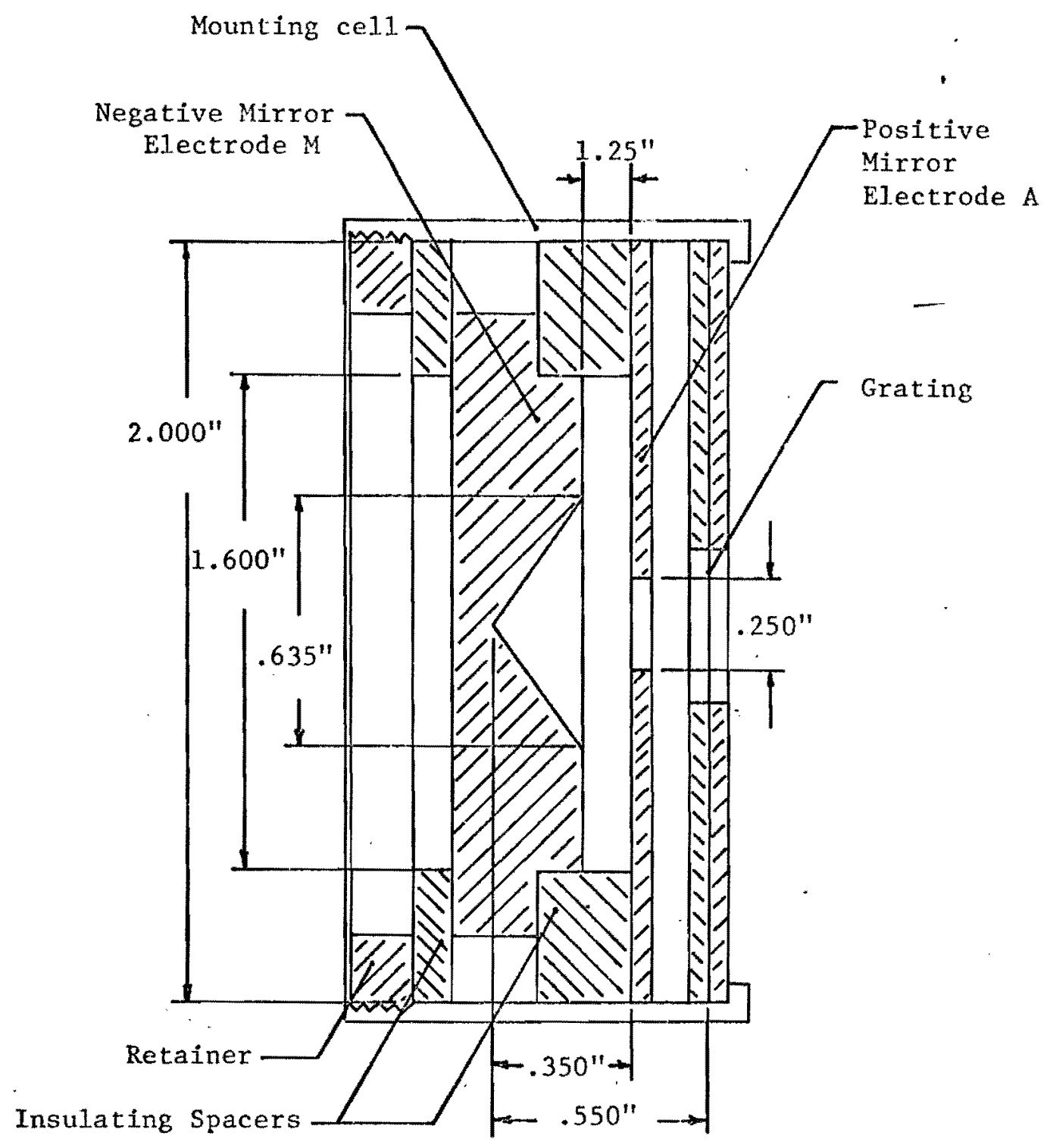

Figure 12. Drawing of the mirror assembly. 
vertex is $u=.550^{\prime \prime}$ and the distance to the plate holder $c+u=$ $6.575^{\prime \prime}$. The grating spacing e is .005".

Sample Data and Experimental Results

To illustrate the method of determining the focal properties of the mirror a numerical example is worked out here for the case where $\gamma^{2}=.962$. The shadow patterns for this case are shown in Fig. 13 where the shadows bear the same relationship to the center of the figure as the wires bear to the optical axis. The shadow patterns In the incident and reflected beams are distinguished by their behavior as the strength of the mirror is changed.

The positions $F$ and $E^{\prime}$ of the shadows cast by the grating lines in the incident and reflected beams are 1isted in Table II. The averages of the shadow positions on opposite sides of the center of the pattern $\bar{E}$ and $\bar{E}^{\prime}$, are also 1isted in Table II. The height of a grating line which corresponds to a shadow cast in the incident beam is given in terms of the number $n$ of grating spacings off axis. Similarly the height of a grating line corresponding to a shadow cast in the reflected beam is specified in terms of $n^{\prime}$. The relationship between $n$ and $n^{\prime}$ is found from the graphs of $\bar{E}$ against $n$ and $\overline{\mathrm{E}}$, against $n^{\prime}$ as shown in Figure 14 .

When the shadow magnifications $M=\bar{E} /$ ne and $M^{\prime}=\bar{E}^{\prime} / n^{\prime}$ e are plotted against $\mathrm{n}^{2}$ the resulting graphs are straight lines according to equations (60) and (61). The sample data are plotted in Figure 15 and lines of estimated best fit are drawn through the data. From the intercepts of these lines the paraxial magnifications are 


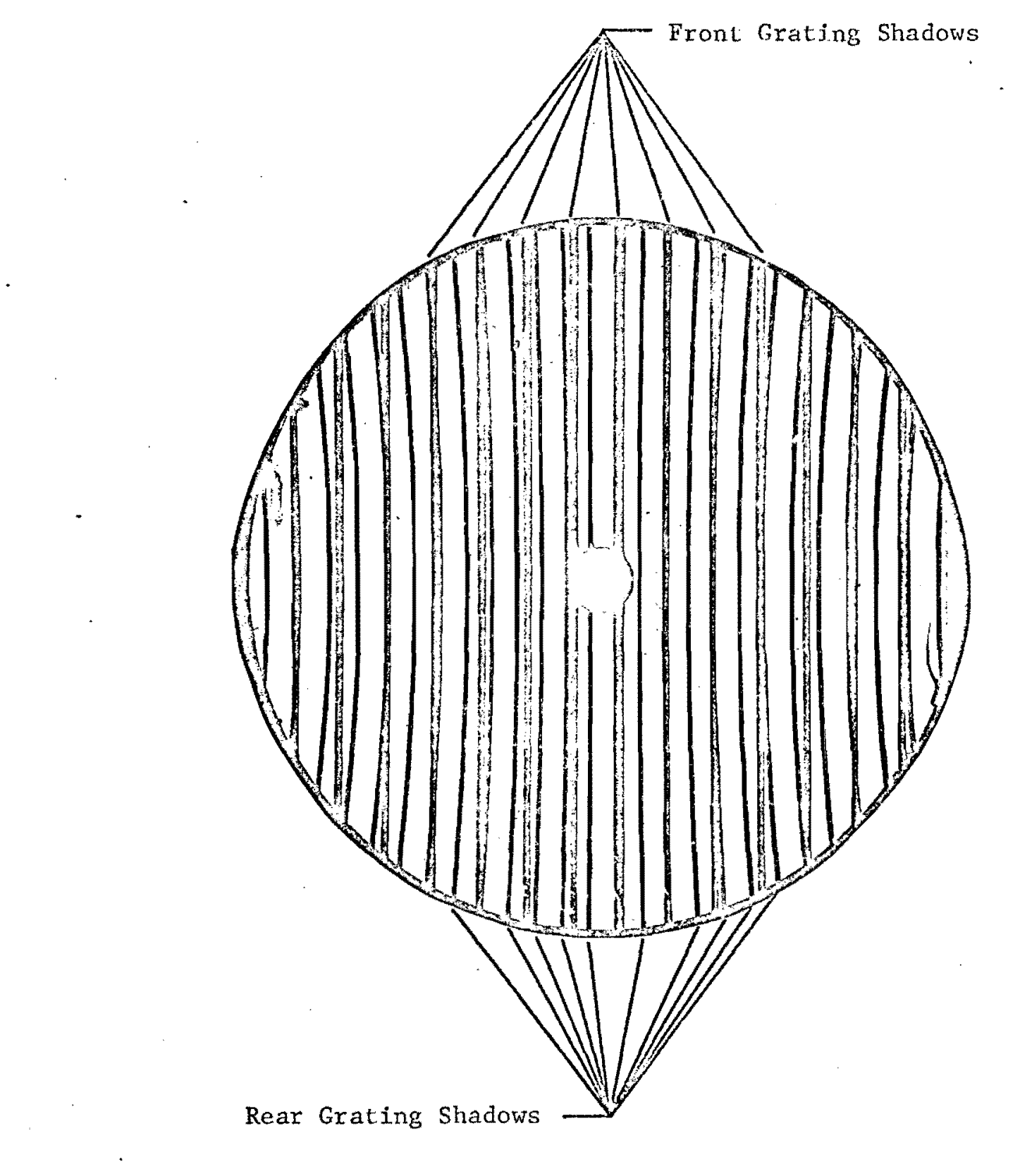

Figure 13. Shadow patterns for the sample data.

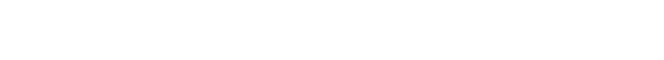


TABLE II

DATA FROM A SHADOW PATTERN

\begin{tabular}{|c|c|c|c|c|c|c|c|c|c|}
\hline $\mathrm{n}$ & $\mathrm{E}(\mathrm{mm})$ & $\overline{\mathrm{E}}$ & $\bar{E} / \mathrm{n}$ & $n^{\prime}$ & $E^{\prime}(\mathrm{mm})$ & $\overline{\mathrm{E}}$ & $\mathrm{n}$ & $\bar{E}^{\prime} / n^{\prime}$ & $\mathrm{n}^{2}$ \\
\hline 0 & 0 & 0 & -- & 0 & 0 & 0 & 0 & -- & 0 \\
\hline 1 & 6.568 & 3.284 & 3.284 & 1 & 3.49 & 1.749 & 0.55 & 1.749 & 0.3 \\
\hline 2 & 13.209 & 6.605 & 3.302 & 2 & 6.968 & 3.484 & 1.05 & 1.742 & 1.1 \\
\hline 3 & 19.909 & 9.955 & 3.318 & 3 & 10.615 & 5.308 & 1.60 & 1.769 & $2 . \epsilon$ \\
\hline 4 & 26.572 & 13.286 & 3.321 & 4 & 14.065. & 7.033 & 2.15 & 1.758 & 4.6 \\
\hline 5 & 32.956 & 16.478 & 3.296 & 5 & 17.628 & 8.814 & 2.70 & 1.763 & 7.3 \\
\hline 6 & 40.272 & 20.136 & 3.356 & 6 & 21.361 & 10.681 & 3.25 & 1.780 & 10.6 \\
\hline & & & & 7 & 24.866 & 12.433 & 3.75 & 1.776 & 14.1 \\
\hline & & & & 8 & 28.848 & 14.424 & 4.35 & 1.803 & 19.0 \\
\hline & & & & 9 & 32.844 & 16.422 & 5.00 & 1.825 & 25.0 \\
\hline & & & & 10 & 36.983 & 18.492 & 5.60 & 1.849 & 31.4 \\
\hline & & & & 11 & 41.278 & 20.639 & 6.25 & 1.876 & 39.0 \\
\hline & & & & 12 & 45.638 & 22.819 & 6.90 & 1.902 & 47.6 \\
\hline
\end{tabular}




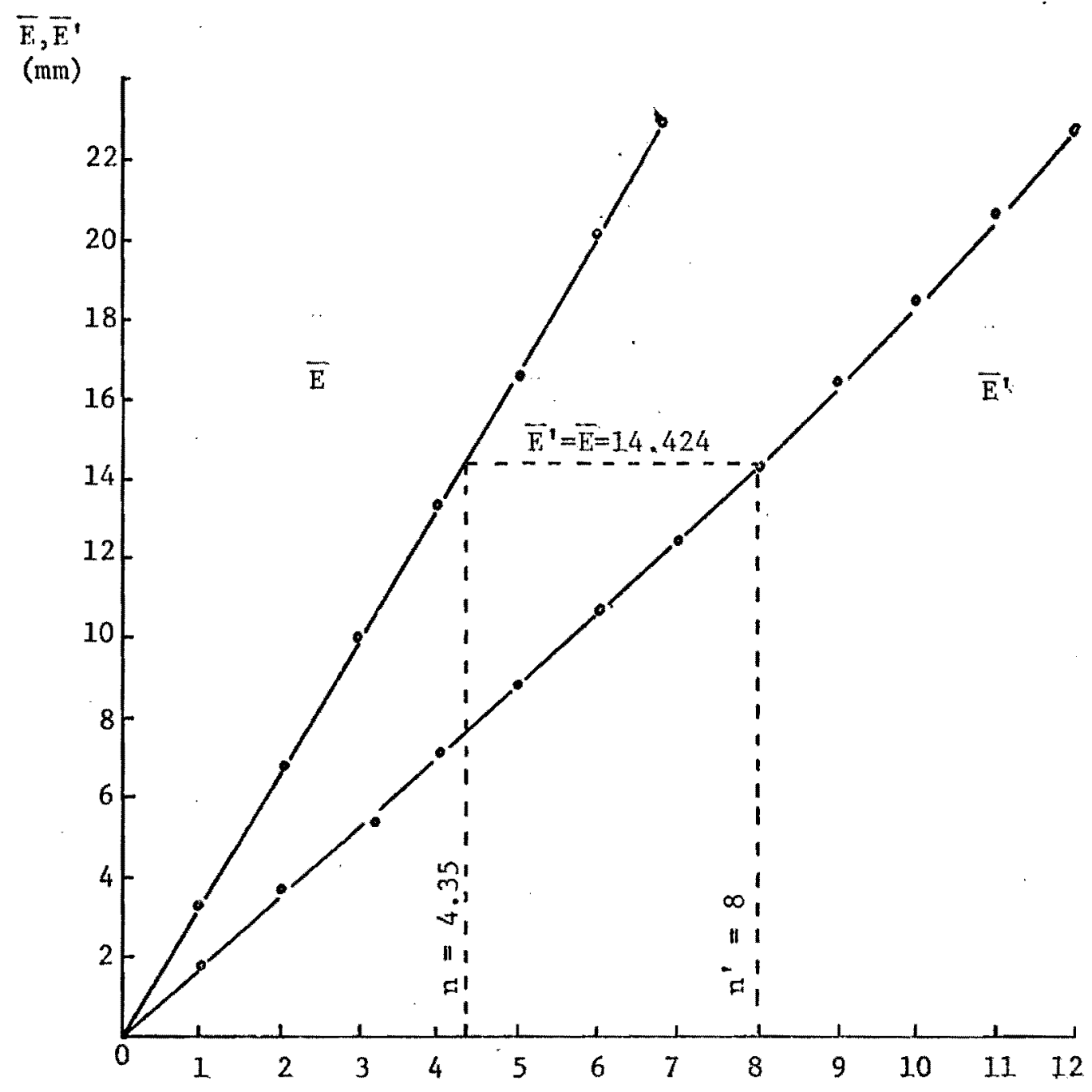

Eigure 14: Method of finding $n$ corresponding to $\overline{\mathrm{E}}^{\prime}$. 


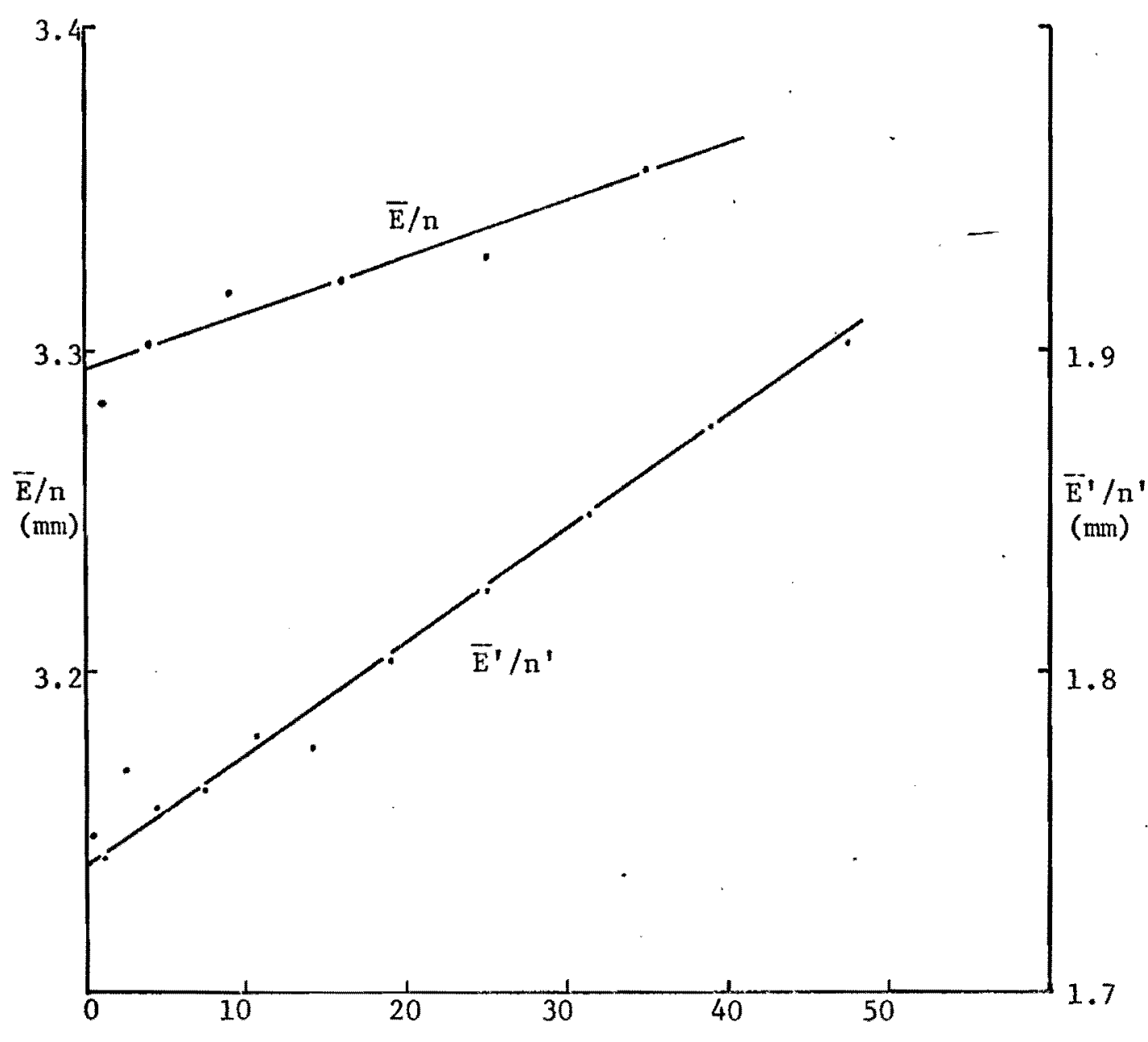

Figure 15. Method of finding the shadow magnifications and distortion coefficients. 


$$
M_{0}=26.0 \pm .1 \quad M_{0}^{\prime}=13.8 \pm .4
$$

and from the slopes the distortions coefficients are

$$
\begin{aligned}
& \beta=(3.0 \pm 1.6) \times 10^{-4} \\
& \beta^{\prime}=(1.9 \pm .3) \times 10^{-3}
\end{aligned}
$$

The grating, source, and recording filn positions are

$$
\begin{aligned}
& u=.550^{\prime \prime} \\
& z=15.265^{\prime \prime} \\
& c=6.575^{\prime \prime}
\end{aligned}
$$

When these values are substituted into equations (64), (65), (68), and (69), the focal properties are computed to be

$$
\begin{aligned}
& f_{0}=.277^{\prime \prime} \pm .002^{\prime \prime} \\
& \mathbf{g}_{0}=.031^{\prime \prime} \pm .016^{\prime \prime} \\
& c_{f}=1.49 \pm .59 \\
& c_{g}=-10.6 \pm 2.4
\end{aligned}
$$

Error is introduced mainly through the nonuniformity of the grating spacings which reduces the precision of $M_{0}, M_{0}{ }^{\prime}, \beta$, and $B^{\prime}$ obtained from the graphs. The physical positions of the various elements are known within $\pm .001^{\prime \prime}$, and the traveling microscope neasurements known within $\pm .002 \mathrm{~mm}$. The reproducibility of the shadow photographs is known to be excellent from other work using this method. The evidence of the nonuniformity of the grating spacings can be seen by examining the similarity of behavior of the individual shadow spacings. The resulting error is estimated 
in the sample calculation but can be calculated by statistical analysis. This nonuniformity becomes a small proportion of the measured distance as the number in of measured grid wires becomes large. The graphical method used here illustrates the method and gives sufficient accuracy for comparison with the theoretically determined focal properties. Where greater accuracy is required the line can be fitted to the data by a least squares method weighted by $n$. Where still more accuracy is required, the grating may be "calibrated" and the individual grating spacing errors compensated. The experimentally determined focal properties are given in Table III for values of $\gamma^{2}$ between .99 and .92 . 
TABLE III

EXPERIMENTAL RESULTS

\begin{tabular}{|c|c|c|c|c|c|c|c|c|}
\hline$\gamma^{2}$ & $B$ & $B^{\prime}$ & $M_{0}$ & $M_{0}^{\prime}$ & $f_{0}$ & $g_{0}$ & $C_{f}$ & $C_{g}$ \\
\hline .990 & .187 & .0818 & 14.3 & 7.88 & .571 & -.445 & 160 & -236 \\
\hline .980 & .0905 & .0649 & 21.4 & 10.3 & .362 & -.176 & 20.8 & -65.2 \\
\hline .971 & .0425 & .0295 & 24.4 & 12.4 & .310 & -.042 & 6.4 & -16.7 \\
\hline .962 & .000304 & .00188 & 26.0 & 13.8 & .277 & .031 & 1.5 & -10.6 \\
\hline .943 & .000200 & .0046 & 25.9 & 15.9 & .287 & .103 & .1 & -1.4 \\
\hline .926 & -.016 & .055 & 24.9 & 18.3 & .285 & .165 & -1.2 & -13.4 \\
\hline
\end{tabular}




\section{CONCLUSIONS}

Graphs of the theoretical and the experimentally determined focal properties of the hyperbolic electron mirror are shown together in Figure 16. Although the curves do not coincide the similarity in behavior with voltage ratio $\gamma^{2}$ is very good. The lack of registration of the two sets of graphs is mainly a result of the relatively large aperture in the positive electrode of the experimental mirror. One of the effects of the large aperture is to reduce the average potential in the mirror field and to increase the time which electrons spend in the mirror field. The result is the same as if the experimental results were shifted to the left along the $\gamma^{2}$ axis. Another effect of the large aperture is a shift In the effective position of the Davisson-Calbick lens associated with the aperture field. This shift results in a larger effective value for the length $z_{A O}$ of the mirror field and hence a longer focal length. The differences between the graphs are much larger than the experimental error and indicate that the net result of the large entrance aperture is an increase in the power of the experimental mirror. It is expected that a hyperbolically shaped positive electrode with a substantially smaller aperture would have properties closer to those of the theoretical model. 
The focal properties of the mirror are given for the range of operation where rays initially parallel to the optical axis cross the axis at most once. This operating range is cailed the first working region. The principle planes are crossed in this region as indicated by the fact that $\mathrm{f}_{0}$ is greater than $g_{0}$. As the mirror electrode is biased closer to the cathode potential, the focal prom perties go to infinity and return in the second working region where rays cross the axis twice. Several other working regions of the mirror were distinguished but not investigated.

The overcorrection of the focal point aberration evident in the graphs in Figure 16 suggests that the mirror could be used to correct the spherical aberration of an election lens which is undercorrected. The compromise made between diffraction and spherical aberration limits the resolution of present electron devices to a few angstroms. Suitable combination of a hyperbolic electron mirror and an objective lens would increase the optimum angular aperture and would thereby make it possible to attain better resolution. The inconvenience of separating incident and reflected beams in such a device has kept this method from being practical so far. 


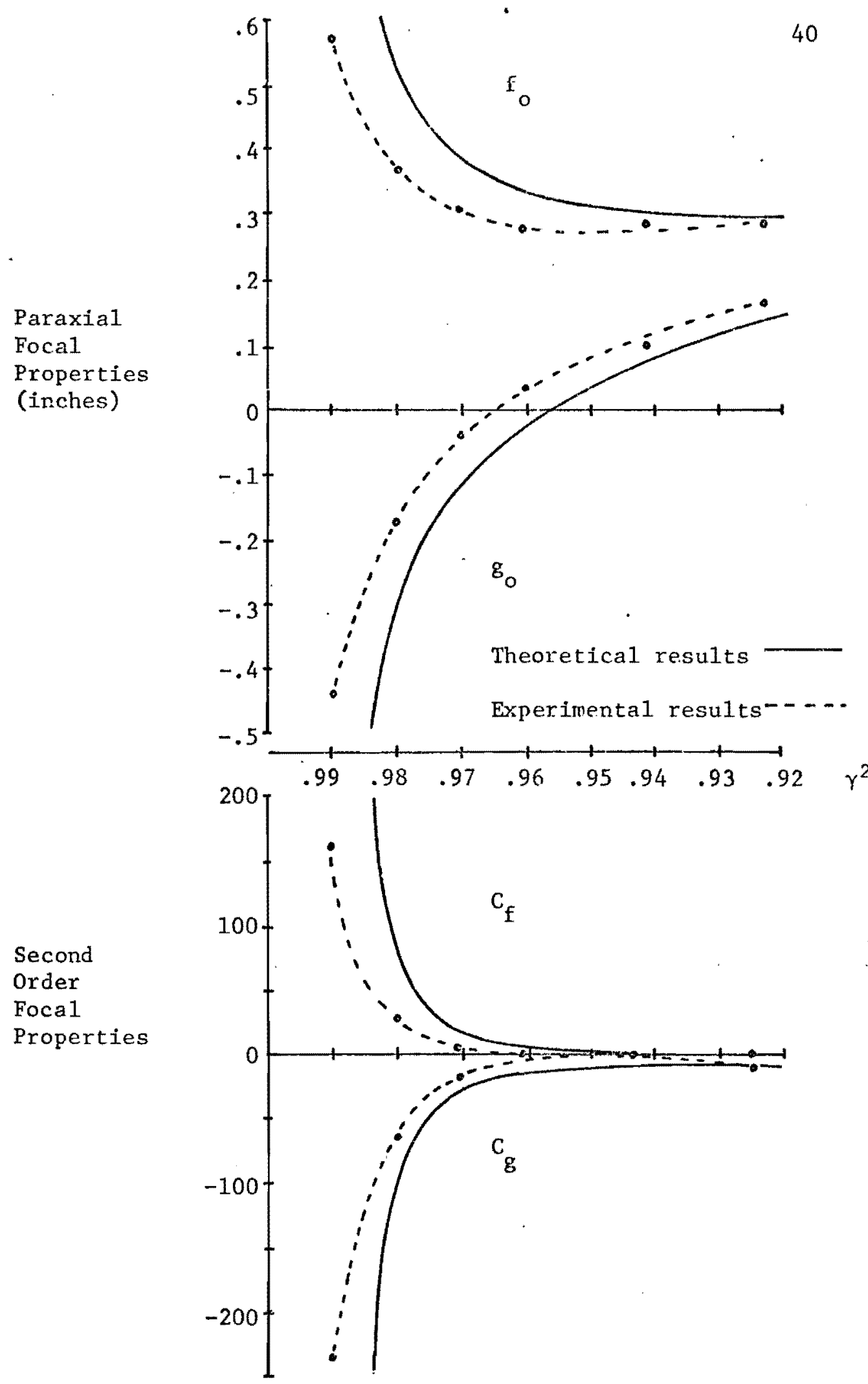

Figure 16. Theoretical and experimental focal properties of the hyperbolic electron mirror. 


\section{BIBLIOGRAPHY}

1. Busch, H., Annalen der Physik, Vol. 81, 9/4-993 (1926)

2. DeProglie, I., Philosophic Magazine, Vol. 47, 446 (1924)

3. Davisson, C. J., and L. H. Germer, Physica1 Review, Vo1. 30, 705 (30 Dec. 1927)

4. Thomson, G. P., Proceedings of the Royal Society, Vo1. 119, 651 (1928)

5. Recknage1, Von A., Zeitschrift fur Physik, Vo1. 104, 381-394 (1936)

6. Hottenroth, Von G., Annalen der Physik, Vol. 30, No. 5, 689-712 (1937)

7. Nico11, F. H., Proceedings of the Physical Society, Vo.1. 50, 888 (1938)

8. Lafferty, J. M., Proceeding of the IRE, Vo1. 35, No. 8, 778-783 (Aug. 1947)

9. Mayer, L., Journal of Applied Physics, Vo1. 26, No. 10, 1228-1230 (Oct. 1955)

10. Rempfer, G. F., Tektronix Research Report on the Electron Mirror Microscope (6 Feb. 1963)

11. Benedict, C., A Simple Electron Mirror, Thesis, Reed College (1963)

12. Jacob, L., An Introduction to Llectron Optics, New York: John Wiley \& Sons, Inc., 15 (1951)

13. Harman, W. W., Electronic Motion, McGraw-Hill, Nery York 37-39 (1953)

14. Davisson, C. J. and C. J. Calbick, Physical. Review, Vo1. 42, 580 (15 Nov. 1932)

15. Spangenberg, K., and I. M. Field, Proceeding of the IRE, vol. 30, 138-144 (Mar. 1942)

16. Rempfer, G. F., Electron Lens Studies, Portland State University (Unpublished) (1966) 
APPENDIX A

THEORETICAL STUDY OF THE MIRROR TO DETERMINE

SECOND ORDER FOCAL PROPFRTIES

The focal length and focal distance and their second order spherical aberrations are obtained in the theoretical study from

$$
\begin{aligned}
& f=r_{A} / \tan \alpha^{\prime} \\
& g=z_{A}^{\prime}+r_{A}^{\prime} / \tan \alpha^{\prime}
\end{aligned}
$$

Relationships ${ }^{1}$ (A1) and (A2) can be expressed in terms of the phase angles $\theta^{\prime}$ and $\phi^{\prime}$ of the exiting electron. From (40), (42), and (46)

$$
\tan \alpha^{\prime}=\frac{\frac{r_{A}^{\prime}}{z_{A}^{\prime}}\left[\frac{\tan \theta^{\prime}}{\sqrt{2} \tanh \phi^{\prime}}-\frac{z_{A}^{\prime}{ }^{2}}{2 \gamma^{2} z_{A O^{2}}^{2}}\right]}{1+\frac{r_{A}^{\prime} \tan \theta^{\prime}}{2 \sqrt{2} \gamma^{2} z_{A O}{ }^{2} \tanh \phi^{\prime}}}
$$

Also $r_{A}^{\prime}=r_{A} \cos \theta^{\prime} / \cos \delta$

$$
=p r_{A}
$$

with $p=\cos \theta^{\prime} / \cos \delta$

1 These and other equations taken from the theoretical study are renumbered here for convenience. 
The expressions for $f$ and $g$ are expressed to second order in $r_{A}$ and separated into paraxial and aberration terms. It is therefore necessary to evaluate the following quantities to second order.

$$
\begin{array}{lr}
\theta^{\prime}=\omega t^{\prime}-\delta & (17),(\mathrm{A} 7) \\
\tan \delta=\frac{-\dot{z}_{A} z_{A}}{2 \omega \gamma^{2} z_{A O}^{2}} & (37),(A 8) \\
\sqrt{2} \omega t^{\prime}=\phi^{\prime}-\varepsilon & \text { [From (18)] (A9) } \\
\cosh \phi^{\prime}=z_{A}^{\prime} \cosh \varepsilon / z_{A} & \text { [From (14)] (A10) } \\
\tanh \varepsilon=-\dot{z}_{A} / \sqrt{2} \omega z_{A} & \text { (12), (A11) }
\end{array}
$$

The quantities $z_{A}, z_{A}$ ', and $\dot{z}_{A}$ expressed to second order, necessary to expand (A7) through (A11), are

$$
z_{A}=z_{A O}\left(1+\sigma^{2}\right)
$$

$$
\begin{aligned}
& \text { with } \sigma=r_{A} / 2 z_{A O} \\
& z_{A}^{\prime}=z_{A O}\left(1+\sigma^{\prime 2}\right)
\end{aligned}
$$

$$
\begin{aligned}
& \text { with } \sigma^{\prime}=\mathrm{r}_{\mathrm{AO}}{ }^{\prime / 2 \mathrm{z}_{\mathrm{AO}}} \\
& \text { or } z_{A}{ }^{\prime}=z_{A O}\left(1+p_{0}^{2} \sigma^{2}\right) \\
& \text { with } \mathrm{p}_{0}=\mathrm{r}_{\mathrm{AO}}{ }^{\prime} / \mathrm{r}_{\mathrm{A}}=\cos \theta_{0}^{\prime} / \cos \delta_{0} \\
& -\dot{z}_{A}=\sqrt{2} \gamma \omega z_{A O}\left(1-\dot{\mathrm{r}}_{\mathrm{AO}}{ }^{2 / 2} \dot{z}_{\mathrm{AO}}{ }^{2}\right)
\end{aligned}
$$


From (34) and (36)

$$
\begin{aligned}
& \frac{\dot{\mathrm{r}}_{\mathrm{A}}}{\dot{-}_{\mathrm{A}}}=\frac{\mathrm{r}_{\mathrm{A}}}{-\mathrm{f}_{\mathrm{A}}}=\frac{\mathrm{r}_{\mathrm{A}}{ }^{z_{A}}}{2 r^{2} z_{\mathrm{AO}}{ }^{2}} \\
& \frac{\dot{\mathrm{r}}_{\mathrm{AO}}}{-\dot{\mathrm{z}}_{\mathrm{AO}}}=\frac{\mathrm{r}_{\mathrm{A}}}{2 \gamma^{2} \mathrm{z}_{\mathrm{AO}}}=\frac{\sigma}{r^{2}}
\end{aligned}
$$

and rewriting (A16) using (A18)

$$
-\dot{z}_{\mathrm{A}}=\sqrt{2} \gamma \omega z_{\mathrm{AO}}\left(1-\sigma^{2} / 2 \gamma^{4}\right)
$$

Substituting (A19) and (A12) into (A11)

$$
\tanh \varepsilon=\tanh \varepsilon_{o}\left(1-\left[1+1 / 2 \gamma^{4}\right] \sigma^{2}\right)
$$

with $\tanh \varepsilon_{0}=\gamma$

Then $\cosh \varepsilon=1 / \sqrt{1-\tanh ^{2} \varepsilon}$

$$
=\cosh \varepsilon_{0}\left[1-\frac{\gamma^{2}}{1-\gamma^{2}}\left(1+\frac{1}{2 \gamma^{4}}\right) \sigma^{2}\right]
$$

with $\cosh \varepsilon_{0}=1 / \sqrt{1-\gamma^{2}}$

The angle $\varepsilon$ is found to second order by comparing (A22) with the expansion

$$
\begin{aligned}
\cosh \varepsilon & =\cosh \left(\varepsilon_{0}+\Delta \varepsilon\right)=\cosh \varepsilon_{0} \cdot 1+\sinh \varepsilon_{0} \cdot \Delta \varepsilon \\
& =\cosh \varepsilon_{0}\left(1+\tanh \varepsilon_{0} \cdot \Delta \varepsilon\right)
\end{aligned}
$$

Then $\Delta \varepsilon=\frac{-\gamma}{1-\gamma^{2}}\left(1+\frac{1}{2 \gamma^{4}}\right) \sigma^{2}$ 
and $\varepsilon=\varepsilon_{0}-\frac{\gamma}{1-\gamma^{2}}\left(1+\frac{1}{2 \gamma^{4}}\right) \sigma^{2}$

Substituting (A.12), (A14), and (A22) into (A10)

$$
\begin{aligned}
\cosh \phi^{\prime} & =\frac{z_{A O}\left(1+p_{0}^{2} \sigma^{2}\right)}{z_{A O}\left(1+\sigma^{2}\right)} \frac{1}{\sqrt{1-\gamma^{2}}}\left[1-\frac{\gamma^{2}}{1-\gamma^{2}}\left(1+\frac{1}{2 \gamma^{4}}\right) \sigma^{2}\right] \\
& =\cosh \phi_{0}^{\prime}\left\{1+\left[p_{0}^{2}-\frac{1+1 / 2 \gamma^{2}}{1-\gamma^{2}}\right] \sigma^{2}\right\}
\end{aligned}
$$

with $\cosh \phi_{0}^{\prime}=1 / \sqrt{1-\gamma^{2}}$

(26) (A28)

Then $\tanh \phi^{\prime}=\sqrt{1-1 / \cosh ^{2} \phi^{\prime}}$

with $\tanh \phi_{0}^{\prime}=\gamma$

$$
=\tanh \phi_{0}\left\{1+\frac{1}{\gamma^{2}}\left[\left(1-\gamma^{2}\right) p_{0}^{2}-\left(1+\frac{1}{2 \gamma^{2}}\right)\right] \sigma^{2}\right\}
$$

The angle $\phi^{\prime}$ is found to second order by comparing (A27)

with the expansion

$$
\begin{aligned}
\cosh \phi^{\prime} & =\cosh \left(\phi_{0}^{\prime}+\Delta \phi^{\prime}\right)=\cosh \phi_{0}^{\prime} \cdot 1+\sinh \phi_{0}^{\prime} \cdot \Delta \phi^{\prime} \\
& =\cosh \phi_{0}^{\prime}\left(1+\tanh \phi_{0}^{\prime} \cdot \Delta \phi\right)
\end{aligned}
$$

Then $\Delta \phi^{\prime}=\frac{1}{\gamma}\left(p_{0}^{2}-\frac{\left(1+1 / 2 \gamma^{2}\right)}{1-\gamma^{2}} \sigma^{2}\right.$

and $\phi^{\prime}=\phi_{0}^{\prime}+\frac{1}{\gamma}\left(p_{0}^{2}-\frac{1+1 / 2 \gamma^{2}}{1-\gamma^{2}}\right) \sigma^{2}$

The time $t$ ' which electrons spend in the mirror field is found to second order by combining (A26) and (A33) with (A9). 
$\omega t^{\prime}=\omega t_{0}{ }^{\prime}+\frac{1}{\sqrt{2} \gamma}\left(p_{0}^{2}-\frac{\gamma^{2}+1+1 / \gamma^{2}}{1-\gamma^{2}}\right) o^{2}$

with the paraxial time $t_{0}$ 'given by

$$
\omega t_{0}{ }^{\prime}=\frac{\phi_{0}{ }^{\prime}-\varepsilon_{0}}{\sqrt{2}}=\sqrt{2} \operatorname{arctanh} \gamma
$$

Substituting (A12) and (A19) into (A8)

$$
\tan \delta=\tan \delta_{0}\left[1+\left(1-\frac{1}{2 \gamma^{4}}\right) \sigma^{2}\right]
$$

with $\tan \delta_{0}=\frac{1}{\sqrt{2} \gamma}$

then $\cos \delta=1 / \sqrt{1+\tan ^{2} \delta}=\cos \delta_{0}\left[1-\left(\frac{1}{2 \gamma^{2}} \frac{1-1 / 2 \gamma^{4}}{1+1 / 2 \gamma^{2}}\right) \sigma^{2}\right]$

with $\cos \delta_{0}=1 / \sqrt{1+1 / 2 \gamma^{2}}$

The ang1e $\delta$ is found to second order by comparing (A38)

with the expansion

$$
\begin{aligned}
\cos \delta & =\cos \left(\delta_{0}+\Delta \delta\right)=\cos \delta_{0}: 1-\sin \delta_{0} \cdot \Delta \delta \\
& =\cos \delta_{0}\left(1-\tan \delta_{0} \cdot \Delta \delta\right)
\end{aligned}
$$

Then $\Delta \delta=\frac{1}{\sqrt{2}}\left[\frac{1-1 / 2}{\gamma} \gamma^{4}\right] \sigma^{2}$

and $\delta=\delta_{0}+\frac{1}{\sqrt{2} \gamma}\left[\frac{1-1 / 2 \gamma^{4}}{1+1 / 2 \gamma^{2}}\right] \sigma^{2}$

The angle $\theta^{\prime}$ is found to second order by substituting (A34) and (A42) into (A7) 


$$
\begin{aligned}
\theta^{\prime} & =\omega t_{0}{ }^{\prime}-\delta_{0}+\frac{1}{\sqrt{2} \gamma}\left[p_{0}{ }^{2}-\frac{5 / 2+2 / \gamma^{2}}{\left(1-\gamma^{2}\right)\left(1+1 / 2 \gamma^{2}\right)}\right] \sigma^{2} \\
& =\theta_{0}^{\prime}+\tau \sigma^{2}
\end{aligned}
$$

with $\theta_{0}^{\prime}=\omega t_{0}^{\prime}-\delta_{0}=\sqrt{2} \operatorname{arctanh} \gamma-\arctan \frac{1}{\sqrt{2} \gamma}$

and $\quad \tau=\frac{1}{\sqrt{2} \gamma}\left[p_{0}{ }^{2}-\frac{5 / 2+2 / \gamma^{2}}{\left(1-\gamma^{2}\right)\left(1+1 / 2 \gamma^{2}\right)}\right]$

The cosine of the angle $\theta^{\prime}$ is found to second order from the expansion

$$
\begin{aligned}
\cos \theta^{\prime} & =\cos \left(\theta_{0}^{\prime}+\Delta \theta^{\prime}\right)=\cos \theta_{0}^{\prime} \cdot 1-\sin \theta_{0}^{\prime} \cdot \Delta \theta \\
& =\cos \theta_{0}^{\prime}\left(1-\tan \theta_{0}^{\prime} \tau \sigma^{2}\right)
\end{aligned}
$$

Then $\tan \theta^{\prime}=\tan \theta_{0}^{\prime}\left(1+\frac{\tau \sigma^{2}}{\cos ^{2} \theta_{0}^{\prime} \tan \theta_{0}^{\prime}}\right)$

The ratio (A6) is now evaluated using (A38) and (A47)

$$
\begin{aligned}
\mathrm{p} & =\mathrm{r}_{\mathrm{A}}^{\prime} / \mathrm{r}_{\mathrm{A}}=\cos \theta^{\prime} / \cos \delta=\frac{\cos \theta_{0}^{\prime}\left(i-\tau \tan \theta_{0}^{\prime} \sigma^{2}\right)}{\cos \delta_{0}\left[1-\frac{1}{2 \gamma^{2}} \frac{\left(1-1 / 2 \gamma^{4}\right)}{\left(1+1 / 2 \gamma^{2}\right)}\right.} \\
& =\mathrm{p}_{0}\left(1+h \sigma^{2}\right)
\end{aligned}
$$

with $h=\frac{1}{2 \gamma^{2}} \frac{\left[1-1 / 2 \gamma^{4}\right]}{\left[1+1 / 2 \gamma^{2}\right]}-\tau \tan \theta_{0}^{\prime}$ 
Finally the angle $\alpha^{\prime}$ of the electron exiting from the mirror is found by substituting $(\Lambda 14),(\Lambda 29)$, and ( 448$)$ into (A3). .

$$
\begin{aligned}
\tan \alpha^{\prime} & =\frac{r_{A}^{\prime}}{z_{A}^{\prime}}\left[\frac{\tan \theta_{0}^{\prime}\left[1+\tau \sigma^{2} /\left(\cos ^{2} \theta_{0}^{\prime} \tan \theta_{0}^{\prime}\right)\right]}{\sqrt{2} \tanh \theta_{0}\left\{1+1 / \gamma^{2}\left[\left(1-\gamma^{2}\right) p_{0}^{2}-\left(1+1 / 2 \gamma^{2}\right)\right] \sigma^{2}\right\}}\right. \\
& \left.-\frac{z_{A O}{ }^{2}\left(1+2 p_{0}^{2} \sigma^{2}\right)}{2 \gamma^{2}{ }_{A O}{ }^{2}}\right] /\left[1+\frac{r_{A}^{2} \cos ^{2} \theta_{0}^{\prime} \tan \theta_{0}^{\prime}}{2 \sqrt{2} \gamma^{2} z_{A O}{ }^{2} \cos ^{2} \delta_{0} \tanh \phi_{0}}\right] \\
& =\frac{1}{\sqrt{2} \gamma}{ }^{{ }^{2}{ }_{A}^{\prime}}\left(\tan \theta_{0}^{\prime}-\tan \delta_{0}\right)\left(1+q \sigma^{2}\right)
\end{aligned}
$$

with

$$
\begin{aligned}
q & =\left\{\frac{\tau}{\cos ^{2} \theta_{0}^{\prime}}-\frac{\tan \theta_{0}^{\prime}}{\gamma^{2}}\left[\left(1-\gamma^{2}\right) p_{0}{ }^{2}-\left[1+\left(1 / 2 \gamma^{2}\right)\right]\right]-\left(\sqrt{2} p_{0}{ }^{2}\right) / \gamma\right. \\
& \left.=\left(\sqrt{2} \mathrm{p}_{0}^{2} \tan \theta_{0}^{\prime}\right) / \gamma^{3}\right\} /\left[\tan \theta_{0}^{\prime}-\tan \delta_{0}\right]
\end{aligned}
$$

The focal length can now be evaluated to second order by substituting (A51) into (A1) and using (A14) and (A49).

$$
\begin{aligned}
f f & =\frac{\sqrt{2} \gamma z_{A}^{\prime} r_{A} / r_{A}^{\prime}}{\left(\tan \theta_{0}^{\prime}-\tan \delta_{0}\right)\left(1+q \sigma^{2}\right)} \\
& =\frac{\sqrt{2} \gamma z_{A 0} / p_{0}}{\left(\tan \theta_{0}^{\prime}-\tan \delta_{0}\right)}\left[1+\left(p_{0}^{2}-q-h\right) o^{2}\right] \\
& =f_{0}\left[1-C_{f}\left(\rho / f_{0}\right)^{2}\right]
\end{aligned}
$$


with the paraxial focal. length

$$
\mathrm{f}_{0}=\frac{\sqrt{2} \gamma^{z_{A O}}}{\mathrm{p}_{0}\left(\tan \theta_{0}-\tan \delta_{0}\right)}
$$

and the aberration of focal length

$$
C_{f}=-\frac{f_{0}^{2}}{4 z_{A O}^{2}}\left(p_{0}^{2}-q-h\right)
$$

where $p$ is the height of the incident ray on the principle plane, here $\rho=\mathrm{r}_{\mathrm{A}}$.

Similarly the focal distance is found to second order by substituting (A14) and (A51) into (A2)

$$
\begin{aligned}
g & =z_{A}^{\prime}+\frac{\sqrt{2} \gamma z_{A}^{\prime}}{\left(\tan \theta_{0}^{\prime}-\tan \delta_{0}\right)\left(1+q \sigma^{2}\right)} \\
& =z_{A O}\left(1+p_{0}^{2} \sigma^{2}\right)+\frac{\sqrt{2} \gamma z_{A O} \frac{\left(1+p_{0}^{2} \sigma^{2}\right)\left(1-q \sigma^{2}\right)}{\left(\tan \theta_{0}^{\prime}-\tan \delta_{0}\right)}}{} \\
& =z_{A O}\left(1+p_{0}^{2} \sigma^{2}\right)+p_{0} f_{0}\left[1+\left(p_{0}^{2}-q\right) \sigma^{2}\right] \\
& =g_{0}-f_{0} C_{g}\left(\rho / f_{0}\right)^{2}
\end{aligned}
$$

with the paraxial focal distance

$$
g_{0}=z_{A O}+\frac{\sqrt{2} \gamma z_{A O}}{\tan \theta_{0}-\tan \delta_{0}}
$$

and the aberration of focal distance

$$
c_{g}=-\frac{f_{0}{ }^{2}}{4 z_{A 0}{ }^{2}} p_{0}\left(p_{0}{ }^{2}-q+\frac{p_{0}{ }^{z} A O}{f_{0}}\right)
$$


APPENDIX B

DERIVATION OF THE EQUATIONS USED IN THE EXPERIMENTAL STUDY

The ray tracing calculations used in the experimental determination of the focal properties of the electron mirror are developed in this section. The expressions for the grating shadow magnifications are inserted into the thick lens expressions for focal length $f$ and focal distance $g$.

The expressions for $f$ and $g$ where image and object space focal properties are the same are taken from the geometrical relationships for the lens or unfolded mirror shown in Figure B1. From similar triangles the magnification $\mathrm{m}$ is

$$
\mathrm{m}=\frac{\mathrm{y}^{\prime}}{\mathrm{y}}=\frac{\mathrm{f}}{\mathrm{z}-\mathrm{g}}=\frac{\mathrm{z}^{\prime}-\mathrm{g}}{\mathrm{f}}
$$

Then $z-g=f / m$

and $z^{\prime}-g=f m$

Subtracting (B2) from (B3) gives

$$
\mathbf{f}=\frac{z-z^{\prime}}{1 / m-m}
$$

and rewritjng (B3) gives

$$
g=z^{\prime}-\mathrm{fm}
$$

In the test apparatus a grid is used to determine the paths of incident and exiting rays as shown in Figure B2. From this figure, the lateral magnification of the source is 


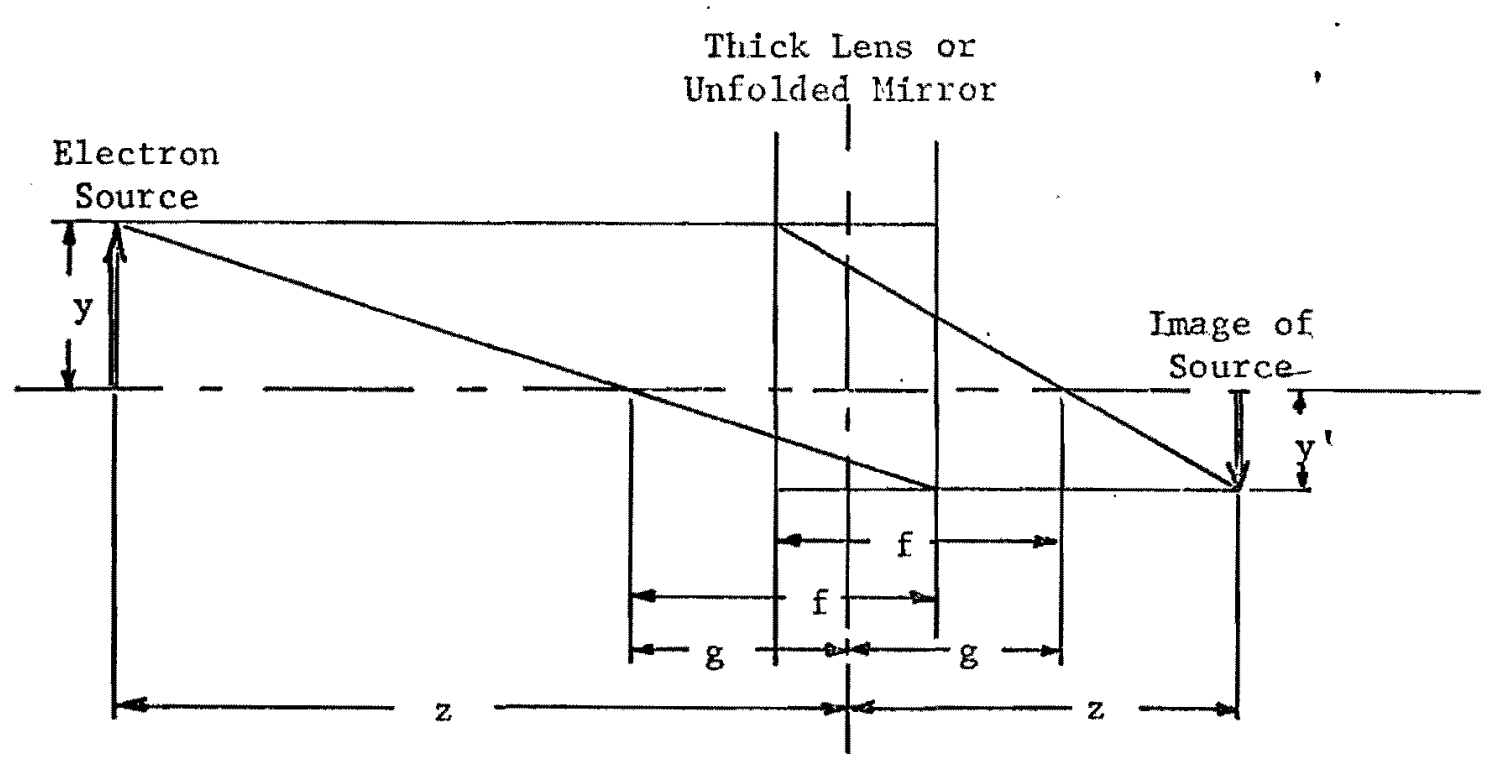

Figure B1. Geometrical relationships of a thick lens.

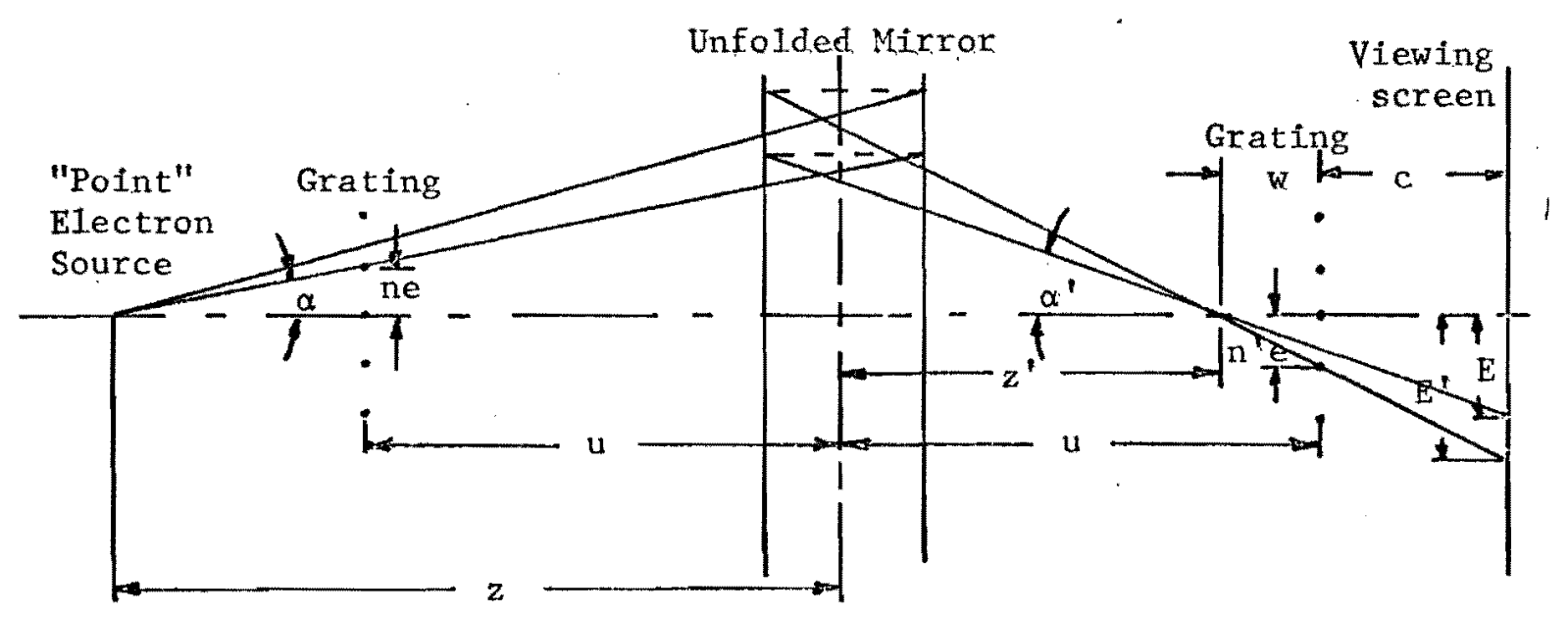

Figure B2. Geometrical relationships of the test apparatus. 


$$
m=\frac{\alpha}{\alpha^{\prime}}=\frac{n e /(z-u)}{E /(w+c)}=\frac{1}{M} \frac{w+c}{z-u}
$$

The distance $w$ is found from the geometric relationship

$$
\frac{W+c}{W}=\frac{E^{\prime}}{n^{\prime} e}=M^{\prime}
$$

or $\quad \mathrm{W}=\frac{c}{M^{\prime}-1}$

The expression for $w$ substituted into $B 6$ gives

$$
m=\frac{M^{\prime}}{M} \frac{1}{M^{\prime}-1} \frac{c}{z-u}
$$

in terms of the fixed distances $c, z$, and $u$, and the measurable shadow magnifications $M$ and $M^{\prime}$. The quantity $z^{\prime}$ is found from the geometric relationship

$$
u-z^{\prime}=w=\frac{c}{M^{\prime}-1}
$$

The shadow magnifications can be expressed with sufficient accuracy as

$$
\begin{aligned}
& M=M_{0}\left(1+\beta n^{2}\right) \\
& M^{l}=M_{0}^{\prime}\left(1+\beta^{\prime} n^{2}\right)
\end{aligned}
$$

where $\mathrm{M}_{0}$ and $\mathrm{M}_{0}$ ' are the paraxial magnification values, $B$ and $B^{\prime}$ are the distortion coefficients, and the height of the incident ray passing through the grating is expressed in terms of the number $\mathrm{n}$ of grating spacings from the axis.

Substituting (B1C) and (B11) into the latera1 magnification (B8) gives

$$
m=m_{0}\left(1-a n^{2}\right)
$$


where

$$
\mathrm{m}_{0}=\frac{\mathrm{M}_{0}^{\prime}}{\mathrm{M}_{0}}-\frac{1}{\mathrm{M}_{0}^{\prime}-1} \frac{\mathrm{c}}{\mathrm{z}-\mathrm{u}}
$$

and

$$
\mathbf{a}=\beta+\frac{\beta^{\prime}}{\mathrm{M}_{0}^{\prime}-1}
$$

The denominator of (B4) is formed from (B12)

$$
\begin{aligned}
& \left.\frac{1}{\mathrm{~m}}-\mathrm{m}=\frac{1}{\mathrm{~m}_{0}}\left(1+a \mathrm{n}^{2}\right)-\mathrm{m}_{0}\left(1-\mathrm{an}^{2}\right)=\frac{1}{\mathrm{~m}_{0}}-\mathrm{m}_{0}+\left(\frac{1}{\mathrm{~m}_{0}}+\mathrm{m}_{0}\right) a \mathrm{n}^{2}(\mathrm{~B}] .5\right) \\
& \frac{1}{\mathrm{~m}}-\mathrm{m}=\left(\frac{1}{\mathrm{~m}_{0}}-\mathrm{m}_{0}\right)\left[1+\frac{1+\mathrm{m}_{0}^{2}}{1-\mathrm{m}_{0}^{2}} \mathrm{an}^{2}\right]
\end{aligned}
$$

Substitution of (B11) into (B9) gives

$$
u-z^{\prime}=w_{0}\left[1-\frac{M_{0}^{\prime} B^{\prime} n^{2}}{M_{0}^{\prime}-1}\right]
$$

with

$$
w_{0}=\frac{c}{M_{0}^{\prime}-1}
$$

The quantity $z^{\prime}$ is obtained from (B17)

$$
\begin{aligned}
& z^{\prime}=z_{0}^{\prime}\left[1-\frac{w_{0}}{z_{0}^{\prime}} \frac{M_{0}^{\prime} \beta^{\prime} n^{2}}{M_{0}^{\prime}-1}\right] \\
& z_{0}^{\prime}=u-w_{0}
\end{aligned}
$$

The numerator of (B4) is formed using (B19)

$$
z-z^{\prime}=\left(z-z_{0}\right)\left[1-\frac{w_{0}}{z-z_{0}^{\prime}} \frac{M_{0}^{\prime} B^{\prime} n^{2}}{M_{0}^{\prime}-1}\right]
$$

and using (B13)

$$
z-z^{\prime}=\left(z-z_{0}^{\prime}\right)\left(1-m_{0} b n^{2}\right)
$$


with

$$
b=\frac{z-u}{z-z_{0}^{\prime}} \frac{M_{0}}{M_{0}^{\prime}-1} B^{\prime}
$$

The focal length (B4) is now formed from (B16) and (B22)

$$
\begin{aligned}
\mathrm{f} & =\frac{\left(z-z_{0}{ }^{\prime}\right)\left(1-\mathrm{m}_{0} b^{2}\right)}{\left[\frac{1}{\mathrm{~m}_{0}}-\mathrm{m}_{0}\right]\left[1+\frac{1+\mathrm{m}_{0}{ }^{2}}{1-\mathrm{m}_{0}{ }^{2}} \mathrm{an}^{2}\right]} \\
& =\mathrm{f}_{0}\left[1-\left[\frac{1+\mathrm{m}_{0}{ }^{2}}{1-\mathrm{m}_{0}{ }^{2}} \mathrm{a}+\mathrm{m}_{0} \mathrm{~b}\right] \mathrm{n}^{2}\right]
\end{aligned}
$$

with the paraxial focal length

$$
f_{0}=\frac{z-z_{0}^{\prime}}{\frac{1}{m_{0}}-m_{0}}
$$

The focal length, expressed in terms of the height $\rho$ of the incident ray at the first principal plane, is

$$
f=f_{0}\left[1-C_{f}\left(\rho / f_{0}\right)^{2}\right]
$$

The height $\rho$ is taken from the geometrical relationship

$$
\frac{p}{z-g+f}=\frac{n e}{z-u}
$$

from which

$$
\frac{\rho}{f}=\left(1+\frac{1}{m}\right) \frac{n e}{z-u}
$$

and

$$
\frac{\rho}{f_{0}}=\left(1+\frac{1}{m_{0}}\right) \frac{n e}{z-u}
$$


Then the aberration coefficient $C_{f}$ is

$$
\begin{aligned}
& \begin{array}{l}
\mathrm{C}_{\mathrm{f}}=\frac{1}{\left[\frac{\mathrm{c}}{\mathrm{z}-\mathrm{u}}\right]^{2}\left[\frac{1}{\mathrm{~m}_{0}}+1\right]^{2}}\left[\frac{1+\mathrm{m}_{0}^{2}}{1-\mathrm{m}_{0}^{2}} \mathrm{a}+\mathrm{m}_{0} \mathrm{~b}\right] \\
\text { The focal distance } \mathrm{g} \text { is found by substituting (B12), } \\
\mathrm{g}=\mathrm{z}_{0} \cdot\left[1-\frac{\mathrm{w}_{0}}{\mathrm{z}_{0}} \frac{\mathrm{M}_{0}^{\prime} \mathrm{B}^{\prime} \mathrm{n}^{2}}{\mathrm{M}_{0}^{\prime}-1}\right]-\mathrm{m}_{0}\left(1-\mathrm{an}^{2}\right) \mathrm{f}_{0}\left[1-\left[\frac{1+\mathrm{m}_{0}^{2}}{1-\mathrm{m}_{0}^{2}} \mathrm{a} \mathrm{m}_{0} \mathrm{~b}\right] \mathrm{n}^{2}\right]
\end{array} \\
& =g_{0}-f_{0}\left[-\frac{2 m_{0}}{1-m_{0}^{2}} a-b\right] n^{2}
\end{aligned}
$$

and (B24) into (B5)

with the paraxial focal distance

$$
g_{0}=z_{0}{ }^{\prime}-m_{0} f_{0}
$$

The focal distance expressed in terms of $p$

$$
\mathbf{g}=\mathbf{g}_{0}-\mathrm{f}_{0} \mathrm{C}_{\mathbf{g}}\left(\rho / \mathrm{f}_{0}\right)^{2}
$$

and the aberration coefficient $\mathrm{C}_{\mathrm{g}}$ is .

$$
c_{g}=\frac{1}{\left[\frac{e}{z-u}\right]^{2}\left[\frac{1}{m_{0}}+1\right]^{2}}\left[-\frac{2 m_{0}}{1-m_{0}^{2}} a-b\right]
$$

Both aberrations (B28) and (B33) can be expressed in image space dimensions as

$$
c_{f}=\frac{1}{\left[\frac{e}{u-z_{0}^{\prime}}\right]^{2}\left[\frac{0}{\left.M_{0}\right]^{2}}\right]^{2}\left(1+m_{0}\right)^{2}}\left[\frac{1+m_{0}^{2}}{1-m_{0}^{2}} a+m_{0} b\right]
$$




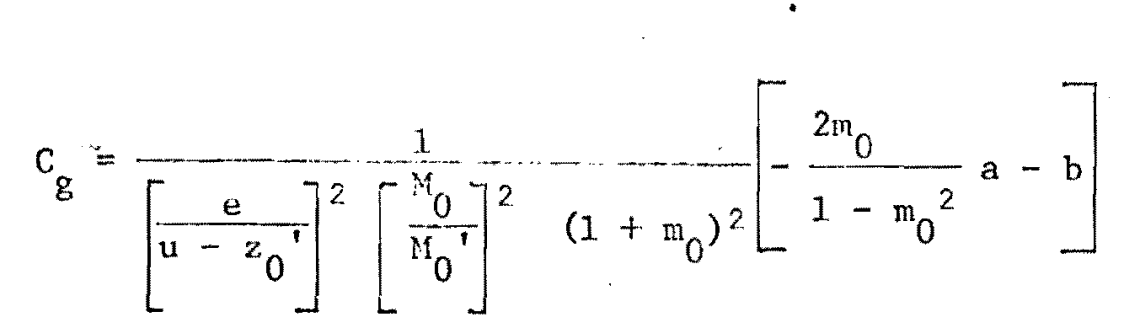

with $a, b$, and $m_{0}$ from (B14), (B23), and (B13). 